SANDIA REPORT

SAND98-0046 • UC-706

Unlimited Release

Printed January 1998
RECEIVEO

FFR 101998

OSTI

\title{
Microholographic Computer Generated Holograms for Security Applications: Microtags
}

William C. Sweatt, Mial E. Warren, Stanley H. Kravitz, Kevin D. Krenz,

Avijit K. Ray-Chaudhuri, Richard H. Stulen, Daniel A. Tichenor, Michael R. Descour

Prepared by

Sandia National Laboratories

Albuquerque, New Mexico 87185 and Livermore, California 94550

Sandia is a multiprogram laboratory operated by Sandia Corporation, a Lockheed Martin Company, for the United States Department of Energy under Contract DE-AC04-94AL85000.

Approved for public release; further dissemination unlimited.

\section{Sandia National Laboratories}

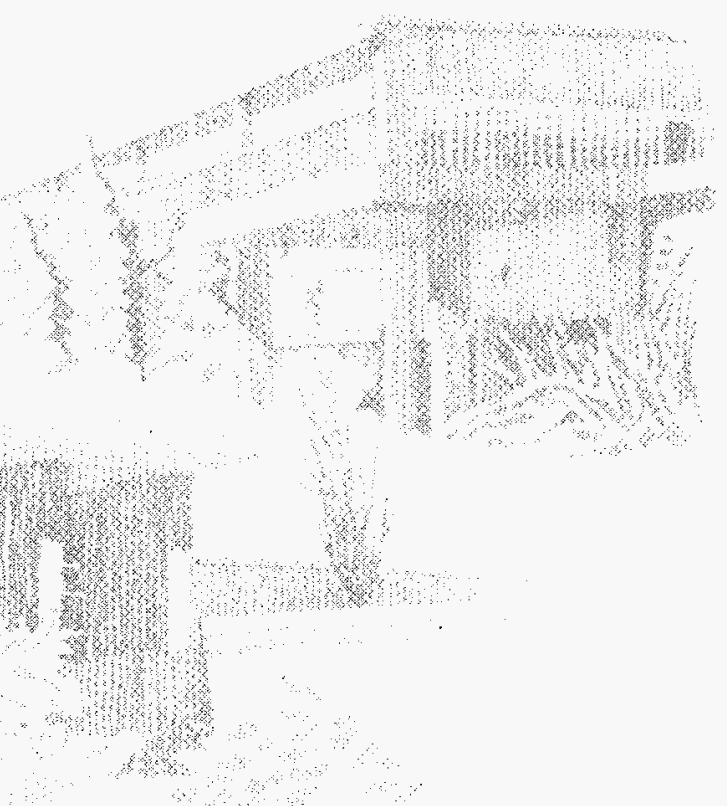


Issued by Sandia National Laboratories, operated for the United States Department of Energy by Sandia Corporation.

NOTICE: This report was prepared as an account of work sponsored by an agency of the United States Government. Neither the United States Government nor any agency thereof, nor any of their employees, nor any of their contractors, subcontractors, or their employees, makes any warranty, express or implied, or assumes any legal liability or responsibility for the accuracy, completeness, or usefulness of any information, apparatus, product, or process disclosed, or represents that its use would not infringe privately owned rights. Reference herein to any specific commercial product, process, or service by trade name, trademark, manufacturer, or otherwise, does not necessarily constitute or imply its endorsement, recommendation, or favoring by the United States Government, any agency thereof, or any of their contractors or subcontractors. The views and opinions expressed herein do not necessarily state or reflect those of the United States Government, any agency thereof, or any of their contractors.

Printed in the United States of America. This report has been reproduced directly from the best available copy.

Available to DOE and DOE contractors from

Office of Scientific and Technical Information

P.O. Box 62

Oak Ridge, TN 37831

Prices available from (615) 576-8401, FTS 626-8401

Available to the public from

National Technical Information Service

U.S. Department of Commerce

5285 Port Royal Rd

Springfield, VA 22161

NTIS price codes

Printed copy: A03

Microfiche copy: A01

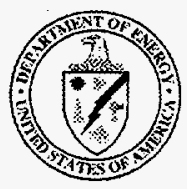




\title{
Microholographic Computer Generated Holograms for Security Applications: Microtags
}

\author{
William C. Sweatt, Mial E. Warren, Photonics Research Department, and Stanley H. Kravitz, \\ Advanced Semiconductor Technology Department, Kevin D. Krenz, \\ Avijit K. Ray-Chaudhuri, Richard H. Stulen, and Daniel A. Tichenor, Advanced Electronics \\ Manufacturing Technology Department, \\ Sandia National Laboratories \\ P.O. Box 5800 \\ Albuquerque, NM 87185-0603 \\ Michael R. Descour, \\ Optical Sciences Center \\ University of Arizona \\ Tucson, Arizona 85721
}

\begin{abstract}
We have developed a method for encoding phase and amplitude in microscopic computer-generated holograms (microtags) for security applications. Eight-by-eight-cell and $12 \times 12$-cell phase-only and phase-and-amplitude microtag designs has been exposed in photoresist using the extreme-ultraviolet $(13.4 \mathrm{~nm}$ ) lithography (EUVL) tool developed at Sandia National Laboratories. Using EUVL, we have also fabricated microtags consisting of 150 -nm lines arranged to form 300 -nm-period gratings. The microtags described in this report were designed for readout at $632.8 \mathrm{~nm}$ and $442 \mathrm{~nm}$. The smallest microtag measures $56 \mu \mathrm{m} \times 80 \mu \mathrm{m}$ when viewed at normal incidence. The largest microtag measures 80 by 160 microns and contains features $0.2 \mu \mathrm{m}$ wide. The microtag design process uses a modified iterative Fourier-transform algorithm to create either phase-only or phase-and-amplitude microtags. We also report on a simple and compact readout system for recording the diffraction pattern formed by a microtag. The measured diffraction patterns agree very well with predictions. We present the results of a rigorous coupled-wave analysis (RCWA) of microtags. Microtags are modeled as consisting of sub-wavelength gratings of a trapezoidal profile. Transverse-electric (TE) and TM readout polarizations are modeled. The objective of our analysis is the determination of optimal microtag-grating design parameter values and tolerances on those parameters. The parameters are grating wall-slope angle, grating duty cycle, grating depth, and metal-coating thickness. Optimal microtag-grating parameter values result in maximum diffraction efficiency. Maximum diffraction efficiency is calculated at $16 \%$ for microtag gratings in air and $12 \%$ for microtag gratings underneath a protective dielectric coating, within fabrication constraints. TMpolarized readout illumination is diffracted with higher efficiency than TE-polarized illumination by microtag gratings. Finally, we suggest several additional microtag concepts, such as twodimensional microtags and pixel-code microtags.
\end{abstract}




\section{Nomenclature}

cell

EUVL

FM

G-S

IFTA

phase-only

phase-and-amplitude

RCWA

ROI

$\eta_{\text {RoI }}$

SNR

SSNR

TE

TM

$\mathrm{T}_{\mathrm{TE}, \text { tookl }}$

$\mathrm{T}_{\mathrm{TM}, \text { total }}$ basic element of microtag, features a complex reflectance Extreme ultraviolet lithography

Folding mirror

Gerchberg-Saxton algorithm, used to design microtags Iterative Fourier-transform algorithm, equivalent to G-S Only phase varies from cell to cell. The modulus of the complex reflectance of each cell is unity across the microtag Phase and amplitude are both allowed to vary across the microtag

Rigorous coupled-wave analysis

Region of interest. Fraction of the far field within which the amplitude of the microtag diffraction pattern is controlled. Feature of the G-S algorithm.

Diffraction efficiency associated with the ROI. Ratio of total irradiance within the ROI to the irradiance in the entire far-field. Signal-to-noise ratio. Used to quantify the fidelity of the microtag diffraction pattern.

Speckle signal-to-noise ratio. Used to quantify irradiance variation within the outline of the letter $E$.

Transverse electric field, relative to plane of incidence

Transverse magnetic field, relative to plane of incidence

Transmittance between air and the protective coating multiplied by the transmittance between the protective layer and air for TEpolarized readout and (-1)st diffraction-order light

Transmittance between air and the protective coating multiplied by the transmittance between the protective layer and air for TM-polarized readout and (-1)st diffraction-order light 


\section{DISCLAIMER}

Portions of this document may be illegible electronic image products. Images are produced from the best available original document. 


\section{Contents}

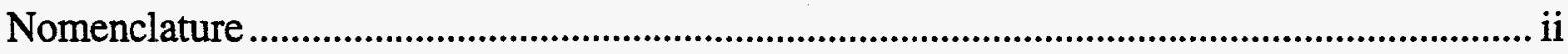

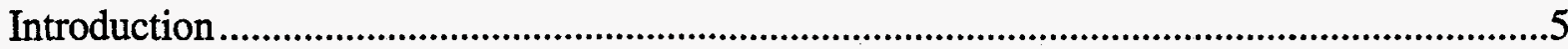

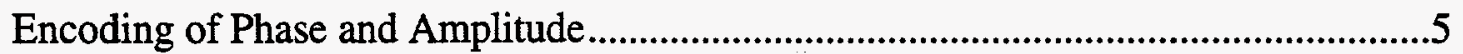

Microtag Design Techniques and Results...................................................................................

Cell-by-cell Perturbation Method .....................................................................................

Improvements with the Perturbation Method .....................................................................10

Microtag Fabrication..............................................................................................................12

Comparison with Alternative Fabrication Techniques ....................................................12

Calculated fabrication tolerances by Rigorous Coupled-Wave Analysis .................................13

Grating Modeling .........................................................................................................13

Rigorous-Coupled-Wave-Analysis Algorithm Performance Verification.....................14

Convergence of the RCWA Algorithm.........................................................................14

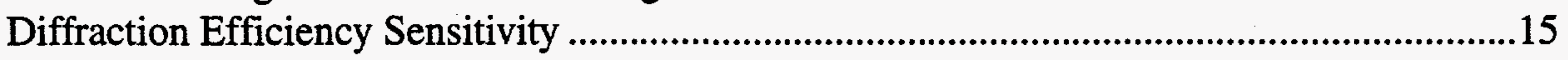

Modeling of a Protective Coating ...............................................................................16

Wall-slope Angle Variation ......................................................................................17

Depth Variation...........................................................................................................18

Duty-cycle Variation ...................................................................................................19

Effect of Metallic Coating Thickness ..........................................................................20

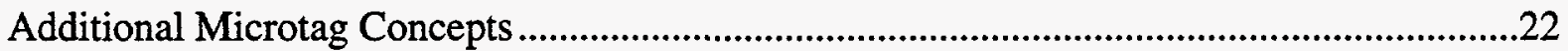

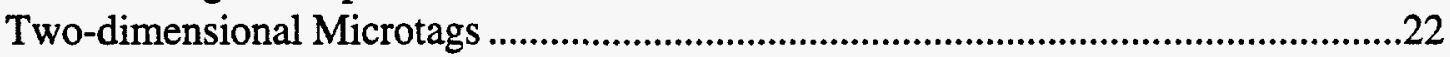

Pixel-code Microtags ....................................................................................................23

"Continuous" Phase-and-amplitude Microtags................................................................23

Practical Implementation .........................................................................................................24

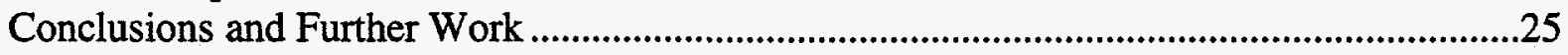

\section{Figures}

$1 \quad$ Phase and amplitude encoding techniques.....................................................................

2 Far-field patterns for the $8 \times 8$ cell phase-and-amplitude microtag................................

$3 \quad$ Far-field patterns for the $8 \times 8$ cell phase-only microtag ...............................................

4 Diffraction patterns from microtags designed with the Gerchberg-Saxton algorithm...9

5 Comparison of predicted and actual diffraction patterns of a $12 \times 12$ cell phase-only microtag

6 Comparison of predicted and actual diffraction patterns of a $8 \times 8$ cell phase-and-

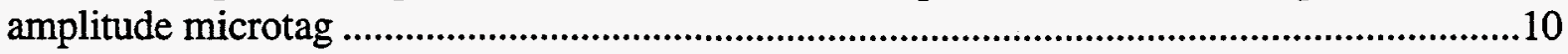

7 Schematic of microtag readout system and phase and amplitude encoding. ................11

$8 \quad$ Portable breadboard readout system ............................................................................12

$9 \quad$ Microtag profile and grating-parameter definition .......................................................13

10 Sensitivity of the (-1)st order diffraction efficiency to grating wall-slope angle .........18

11 Sensitivity of the (-1)st order diffraction efficiency to grating groove depth...............19

12 Sensitivity of the (-1)st order diffraction efficiency to grating duty cycle, $f$...............20

13 Effect of metal-coating thickness on read-out diffraction efficiency ..............................21

14 Predicted diffraction pattern for a 2D phase-only microtag............................................22 
15 Predicted diffraction patterns for a "pixel-code" phase-and-amplitude microtag.........23

16 Predicted diffraction pattern for a 120 phase-quantization level and 40 amplitudelevel microtag

\section{Tables;}

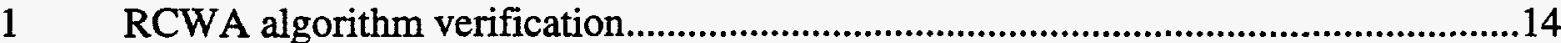

2 Grating parameters used in the RCWA convergence studies ..................................15

3 Microtag parameter nomenclature and variation ranges ...............................................15

$4 \quad$ Nominal grating parameters.........................................................................................16

$5 \quad$ Air-protective-coating interface transmissivities. ............................................................17

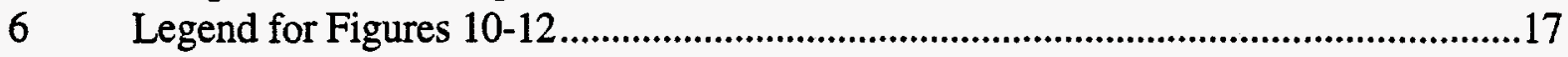

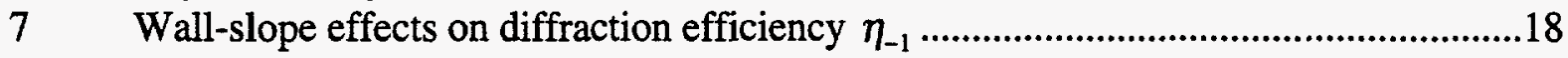

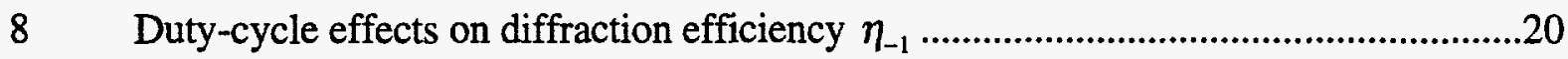

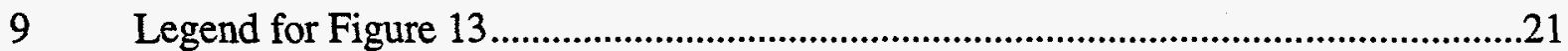




\section{Microholographic Computer Generated Holograms for Security Applications: Microtags}

\section{Introduction}

The microtag concept is an anti-counterfeiting and security measure. ${ }^{1}$ Microtags may be embedded in integrated circuits, on smart cards, ${ }^{2}$ on credit cards, on passports, and on compact discs (CDs). Microtags measure 80 by 160 microns when viewed at normal incidence. The size of the features that comprise the microtag is $0.2 \mu \mathrm{m}$ and can be made as small as $0.075 \mu \mathrm{m} .{ }^{3}$ For comparison, the width of a pit on a CD is $0.5 \mu \mathrm{m} .{ }^{4}$ Microtags are virtually invisible to the naked eye. Microtags' resistance to counterfeiting stems from the uniqueness of the capability to fabricate sub- $0.2-\mu \mathrm{m}$ features.

Microtags are binary structures. This means that only one mask and one exposure is needed to fabricate a complete microtag [see Fig. 1(a)]. We use the following nomenclature: a microtag consists of a set of cells, arranged on a rectangular grid. Each cell is a grating with a $0.4-\mu \mathrm{m}$ grating period. Furthermore, each cell is characterized by a complex reflectance, i.e., an amplitude and a phase. Formally stated, this complex reflectance is $\left|\rho_{m n}\right| \exp \left(j \varphi_{m n}\right)$, where $m$ and $n$ are indices that denote the cell's location within the microtag. The cells and thus the microtags are rectangular when viewed at normal incidence and appear square when observed at the readout angle-of-incidence. For example, the cells have an aspect ratio of 2 when read out at an angle of incidence of $60^{\circ}$ [see Fig. 1(b)].

The term phase-only means that the reflectance amplitude, $\left|\rho_{m n}\right|$, does not vary across the microtag. Information is encoded in the microtag hologram only by the phase values associated with each cell, $\varphi_{m n}$. The term phase-and-amplitude means that the reflectance amplitude is also utilized in encoding information. The additional use of the reflectance amplitude reduces the squared error between the desired and actual irradiance distributions in the far field. On the other hand, the use of the reflectance amplitude also decreases the average reflectance of the microtag, thus reducing the radiance in the diffracted beam.

\section{Encoding of Phase and Amplitude}

The phase at each cell is encoded by a lateral shift of that cell's grating. ${ }^{1}$ In principle, the technique is similar to the detour-phase encoding of Brown and Lohmann. ${ }^{5}$ The schematic in Fig. 1(a) shows several examples of the encoding method. The gratings in each cell are translated left or right by a fraction of the grating constant. The minimum phase increment is determined by the positioning accuracy of the commercial electron-beam writer used to fabricate the 10 -fold magnified microtag photomask. That positioning accuracy is $0.1-\mu \mathrm{m}$ and given a $4-\mu \mathrm{m}$ grating period at the photomask level, such accuracy results in 40 equally spaced phase-quantization levels. In the case of second-generation microtags with 300 -nm period gratings, the $0.1-\mu \mathrm{m}$ positioning accuracy results in 30 equally spaced phasequantization levels. A similar technique for phase-encoding has been developed by Li, et al. ${ }^{6}$ 


\section{Output
beam}

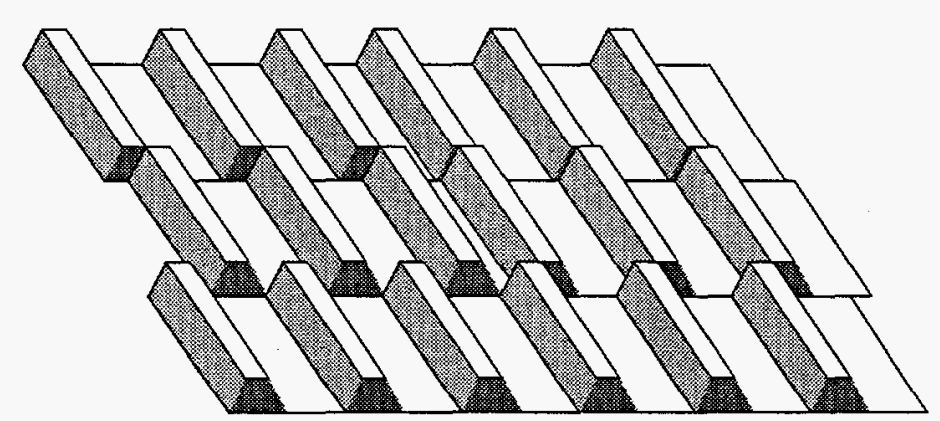

(a)

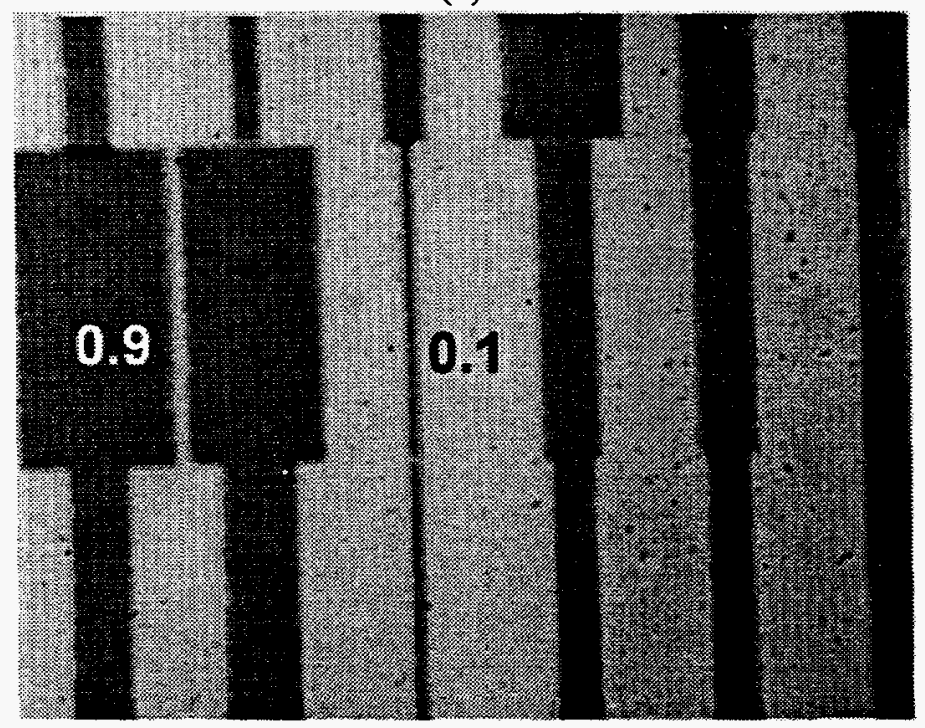

(b)

Figure 1. Phase and amplitude encoding techniques, (a) and (b) respectively. In Part (b), the grating lines are horizontal. See text for details.

Reflectance-amplitude encoding is illustrated in Fig. 1(b). The width of the grating within each cell is trimmed from its full width of $10 \mu \mathrm{m}(|\rho|=1)$ to a minimum of $1 \mu \mathrm{m}$ $(|\rho|=0.1)$ in $1-\mu \mathrm{m}$ decrements according to the required reflectance. The maximum reflectance amplitude shown in Fig. 1(b) is $|\rho|=0.9$ and the minimum $|\rho|=0.1$. Intermediate reflectance-amplitude values are also shown but not marked. An alternative approach to encoding $|\rho|$ requires changes in the grating duty cycle. ${ }^{6}$ The use of the reflectance amplitude improves the diffraction pattern's fidelity, but it decreases the average reflectance of the microtag, so that the diffraction pattern appears dimmer. For example, the average reflectance of a $8 \times 8$ cell phase-and-amplitude microtag is $62 \%$ lower than the average reflectance of a $8 \times 8$ cell phase-only microtag. 
Figures 3(b) and 4(b) illustrate the diffraction patterns formed by microtags fabricated with the EUVL tool. These diffraction patterns match their calculated counterparts extremely well [compare Figs. 3(a) and 3(b) and Figs. 4(a) and 4(b)]. The agreement is satisfactory in terms of the reproduction of the letter "E" and the reproduction of the speckle patterns.

\section{Microtag Design Techniques and Results}

Microtags are designed using the Gerchberg-Saxton (G-S) algorithm, also known as the Iterative Fourier-Transform algorithm (IFTA). ${ }^{7}$ Uniform illumination over the entire microtag is assumed. From a practical viewpoint, this means that the read-out beam's diameter must be large relative to the dimensions of a microtag. The starting point for this iterative algorithm consists of the desired far-field amplitude distribution (in the shape of the letter "E," for instance) and a uniformly random phase distribution in the far field.

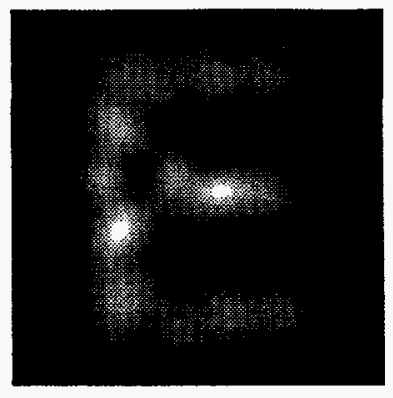

(a)

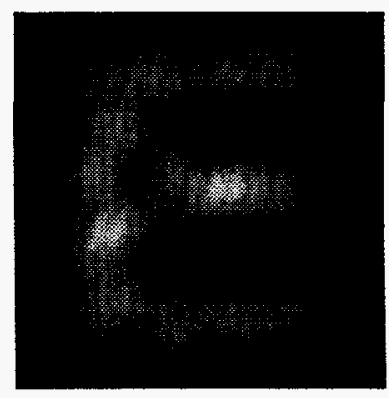

(b)

Figure 2. Far-field patterns for the $8 \times 8$ cell phase-and-amplitude microtag. The simulated far-field pattern is shown in (a). The far-field diffraction pattern formed by a real microtag is shown in (b). Both images have been adjusted in terms of brightness and contrast settings for print quality. The image in (b) has also been rotated to counter a misalignment in the readout system.

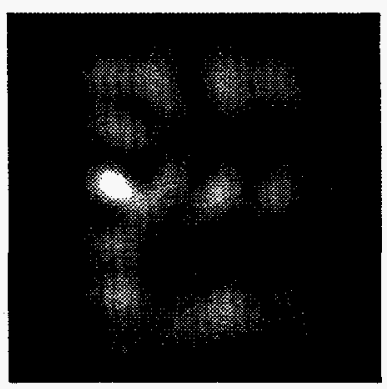

(a)

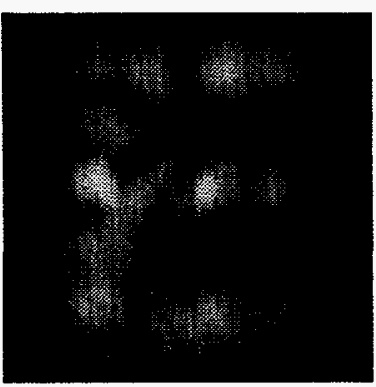

(b)

Figure 3. Far-field patterns for the $8 \times 8$ cell phase-only microtag. The simulated far-field pattern is shown in (a). The measured diffraction pattern formed by a real microtag is shown in (b). 
After each algorithm iteration, the phase and amplitude at each microtag cell were adjusted to the nearest of 40 phase values and 10 amplitude values. The 40 phase values are the maximum number of phase values available in the case of a microtag consisting of $400-\mathrm{nm}$ period gratings. Figures 2(a) and 3(a) show the calculated diffraction patterns to which the algorithm converged after 30 iterations for the phase-and-amplitude and the phase-only cases, respectively.

\section{Cell-by-cell Perturbation Method}

The initial design using the G-S algorithm can be followed by a cell-by-cell perturbation method designed to increase the signal-to-noise ratio (SNR) of the far-field diffraction pattern. The diffraction efficiency of the microtag, defined below, is not used to guide the perturbation method. We define the SNR as $\sum_{i=1}^{M} E_{i} / \sqrt{\sum_{i=1}^{M}\left(\alpha \hat{E}_{i}-E_{i}\right)^{2}}$, where $E_{i}$ is the desired irradiance at the $i$ th pixel in the far field, $\hat{E}_{i}$ is the microtag diffractionpattern irradiance at the $i$ th pixel in the far field, and $M$ is the number of pixels that constitute the region of interest (ROI) in the far field. Furthermore, the scaling factor $\alpha$ is defined as $\sum_{i=1}^{M} E_{i} \hat{E}_{i} / \sum_{i=1}^{M} \hat{E}_{i}^{2}$ and the diffraction efficiency, $\eta_{R O I}$, is defined as $\sum_{i=1}^{M} \hat{E}_{i} / \hat{E}_{t o t}$, where $\hat{E}_{\text {tot }}$ is the total irradiance in the far field in the $(-1)^{\text {st }}$ diffraction order.

The design-algorithm starts with a random phase distribution which is Fourier transformed to the far-field. Next the amplitude distribution within the far-field region of interest is replaced with the desired amplitude distribution (a block letter E). After inverse transforming the modified pattern, the phase and amplitude at each microtag cell are quantized to the nearest of 30 phase values and 11 amplitude values, including zero. Any non-zero phase and amplitude values outside of the $N \times N$ cell microtag are discarded. After 25 iterations of the Gerchberg-Saxton algorithm the result is passed on to the perturbation algorithm. We have found that beyond 25 iterations the SNR ceases to improve. For example, the SNR for the $12 \times 12$ cell microtag increased an average of only $1.4 \%$ after an additional 75 iterations with the Gerchberg-Saxton algorithm. Figure 4 shows the diffraction patterns that would result from using only the Gerchberg-Saxton algorithm for 25 iterations. Note in Figure 4(b) the smoothing effect that occurs when the amplitude is allowed to vary. This effect is even more noticeable when comparing the predicted patterns after the perturbation scheme has been employed, Figure 5(a) and Figure 6(a). It is also worth noting the smaller speckle size in Fig 2(a) relative to 2(b) which is a direct result of the greater surface area of the $12 \times 12$ cell microtag. The speckle size and the microtag area are inversely related through the Fourier transform, and since each cell is the same size, the $12 \times$ 12 cell tag is $125 \%$ larger in area than the $8 \times 8$ cell tag. 


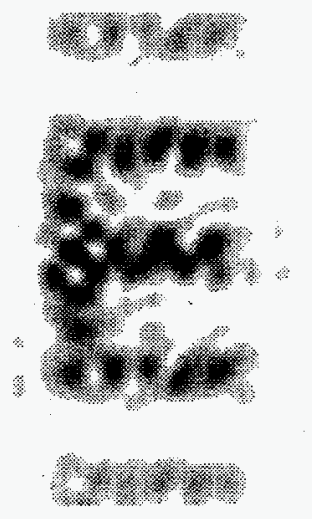

(a)

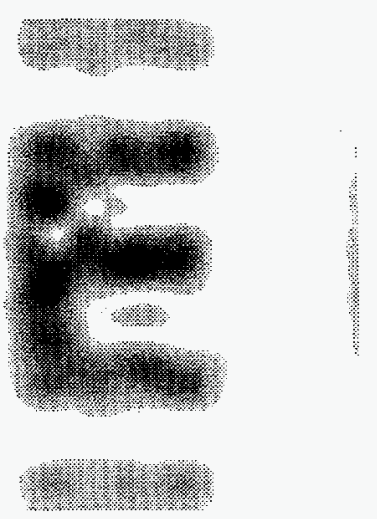

(b)

Figure 4. Diffraction patterns from microtags designed with the Gerchberg-Saxton algorithm. Part (a) shows the predicted diffraction pattern of a $12 \times 12$ cell phase-only microtag designed with 25 iterations of the Gerchberg-Saxton algorithm. Part (b) shows the predicted diffraction pattern of an $8 \times 8$ cell, phase-and-amplitude microtag designed with 25 iterations of the Gerchberg-Saxton algorithm.

In the perturbation algorithm, each cell has an integer multiple of the phasequantization step, $k$ and $\Delta \phi$, respectively, randomly added to or subtracted from the current phase value, $\phi_{m n}$. The indices $m$ and $n$ identify a cell within the microtag. The SNR of the perturbed diffraction pattern is calculated. If the new SNR is greater than the previously calculated SNR, the cell is assigned the perturbed phase value, $\phi_{m n}^{\prime}=\phi_{m n} \pm k \Delta \phi$; otherwise no change is made and the next cell is perturbed. The algorithm can step through the entire microtag more than once for each $k \Delta \phi$ perturbation, and the value of the factor $k$ can be altered, that is, usually reduced, during consecutive passes. For a phase-and-amplitude microtag the procedure is repeated, this time increasing or decreasing the amplitude reflectance, $|\rho|$, of each cell by an integer multiple of the amplitude-quantization step. Increasing or decreasing $|\rho|$ of a cell is equivalent to trimming or widening the grating contained in that cell (see Fig. 7). The amplitude reflectance is confined to the range $0 \leq|\rho| \leq 1$. Only changes which increase the SNR are kept. The combined use of the Gerchberg-Saxton technique followed by a perturbation technique as described above has been previously introduced by Keller and Gmitro as preconditioned random search. ${ }^{8}$ We have simply extended its use to design phase-and-amplitude computer-generated holograms. 


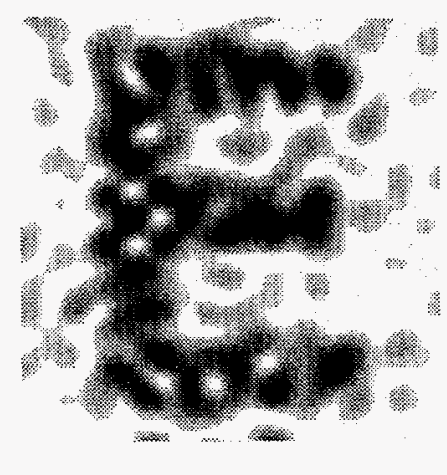

(a)

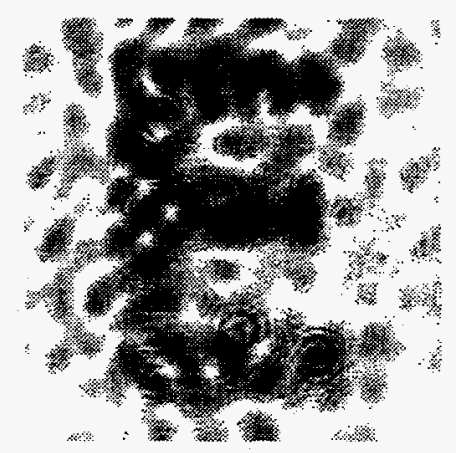

(b)

Figure 5. Comparison of predicted and actual diffraction patterns of a $12 \times 12$ cell phaseonly microtag. Part (a) shows an inverted image of the predicted diffraction pattern. Part (b) shows an inverted image of the actual diffraction pattern.

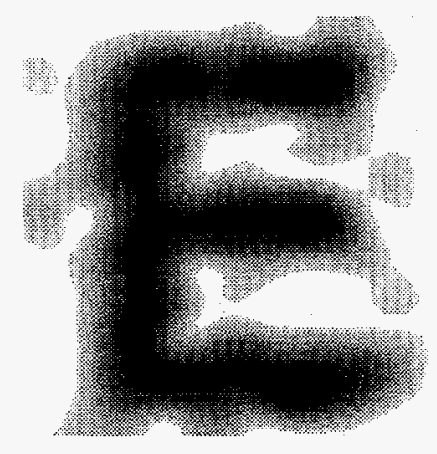

(a)

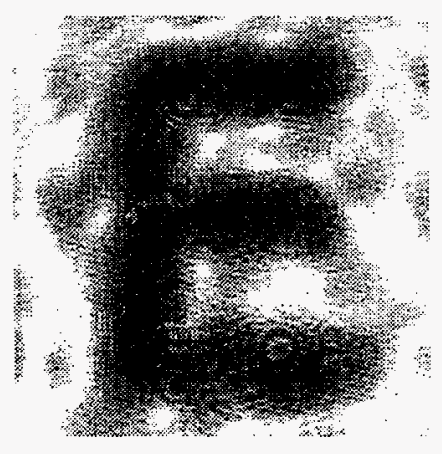

(b)

Figure 6. Comparison of predicted and actual diffraction patterns of a $8 \times 8$ cell phase-andamplitude microtag. Part (a) shows an inverted image of the predicted diffraction pattern. Part (b) shows an inverted image of the actual diffraction pattern.

\section{Improvements with the Perturbation Method}

The SNR is a seemingly rough measure of image quality and the perturbation scheme is quite simple, but there is a significant improvement in the fidelity of the diffracted pattern after using it-compare Figure 4 with Figure 5(a) and 6(a). Note how after applying the perturbation algorithm the diffracted light is more evenly distributed while in the GerchbergSaxton pattern most of the light is confined to the central portion of the diffraction pattern. To quantify the improvement, we define a speckle signal-to-noise ratio (SSNR) as the average irradiance value of the CGH far-field diffraction pattern within the region defined by the outline of the ideal letter $\mathbf{E}$, divided by the standard deviation of the irradiance values contained in that same region. For the phase-only microtags in Figure 5 the SSNR of the perturbed pattern is $26 \%$ greater than it is for the Gerchberg-Saxton pattern. In comparison, 
for the phase-and-amplitude microtags in Figure 6 the SSNR of the perturbed pattern is 55\% greater than it is for the pattern using only the Gerchberg-Saxton algorithm.

\section{Microtag Readout System}

The modulus squared of the Fourier transform of the microtag's complex amplitude reflectance is observed at a distance $z$ measured from the microtag if the Fraunhofer condition on $z$ is satisfied. That condition depends on the maximum radial extent of the microtag, $L_{r}$, and the read-out wavelength, $\lambda: z \gg \pi L_{r}^{2} / \lambda .{ }^{9}$ In the case of the microtags discussed in this report, the values $L_{r}=57 \mu \mathrm{m}$ and $\lambda=0.6328 \mu \mathrm{m}$ lead to the requirement that $z \gg 16$ $\mathrm{mm}$. In practical terms, a separation of $90 \mathrm{~mm}$ between the microtag and an imaging detector array was found to be sufficient to form the microtag's Fourier transform on the detector array without any intervening optics (see Figure 7).

Microtags must be read out at a large angle of incidence measured with respect to the microtag normal. Only then do the $0.4-\mu \mathrm{m}$-period microtag gratings support a diffraction order. Otherwise, we only observe a specular reflection $\left(0^{\text {th }}\right.$ diffraction order $)$. The $0.4-\mu \mathrm{m}-$ period-grating microtags discussed in this report were read out using a $0.5-\mathrm{mW}$ HeNe laser $(0.6328 \mu \mathrm{m})$ incident at $60^{\circ}$ and the resultant diffraction patterns were detected with a $2 / 3$ "format CID camera [see Figs. 2(b), and 3(b)].

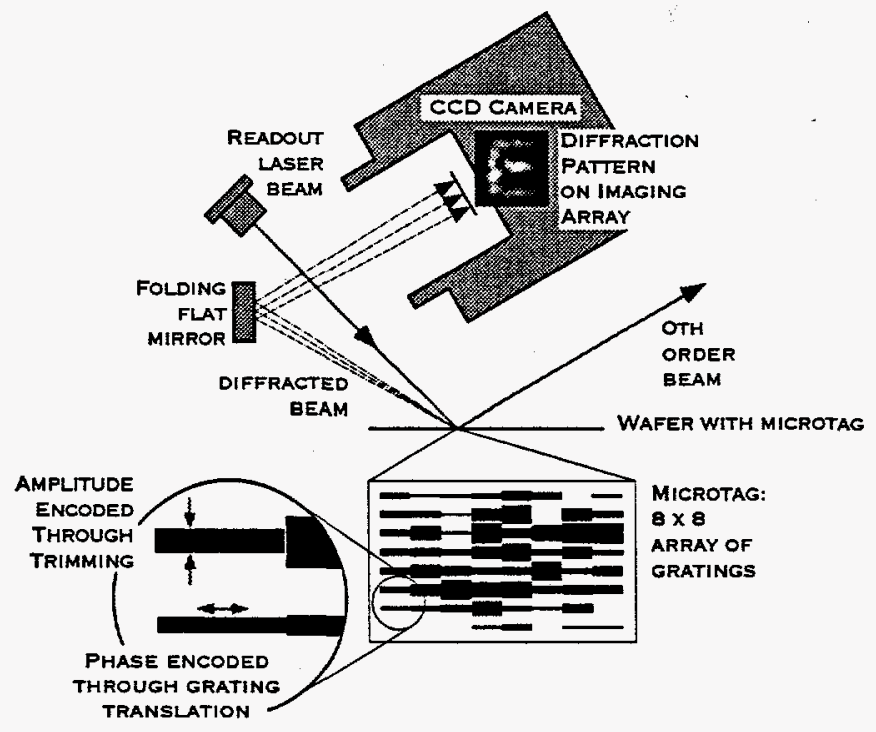

Figure 7. Schematic of microtag readout system and phase and amplitude encoding.

A portable breadboard readout system was constructed to allow the illumination of microtags with a variety of sources. For instance, 300 -nm-period grating microtags were read out with a HeCd laser (442 nm) located in a clean-room facility at the Optical Sciences Center, University of Arizona. We coupled the HeCd laser beam into the readout system via a fold mirror, placed where the laser source is shown in Fig. 7. The readout beam was incident on the microtag at a $42^{\circ}$ angle. Figure 8 shows a top view of the readout-system 
components. The portable readout system was constructed on a $22 \times 28 \mathrm{~cm}^{2}$ breadboard. The optics were contained in a volume measuring approximately $16 \times 13 \times 12 \mathrm{~cm}^{3}$.

The readout system is itself very simple. The readout system consists of a laser source, either integrated (see Fig. 8) or external, a lens to focus the beam waist on the microtag, the microtag itself, and an imaging array to capture the diffracted image. Figure 8 shows a 635-nm laser diode as the illumination source. Both the laser-diode source and the camera shown in Fig. 8 are powered by batteries. In addition, the camera features a liquidcrystal display (LCD). This feature allows for direct viewing of the diffraction pattern formed by the microtag. Although the readout system shown in Fig. 8 is only a breadboard arrangement, we suggest that it contains all the components that would be present in a specialized and ruggedized readout system.

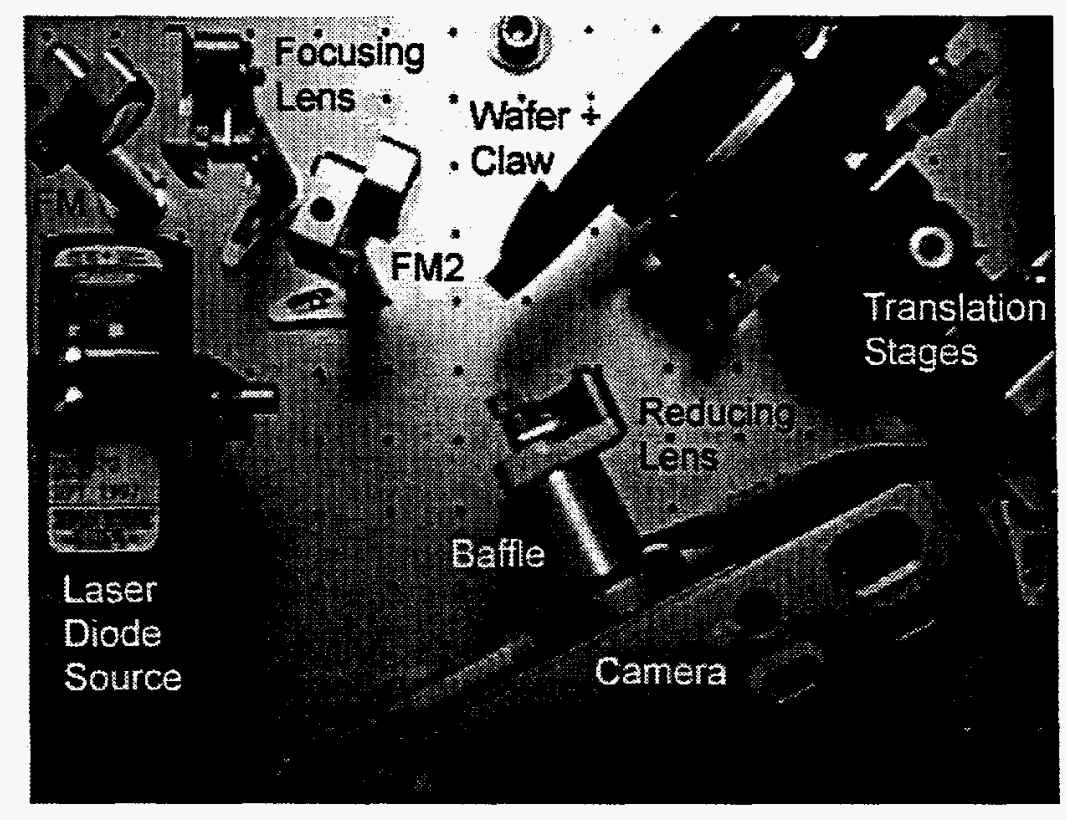

Figure 8. Portable breadboard readout system. FM: folding mirror.

\section{Microtag Fabrication}

Microtags were fabricated on resist-coated silicon wafers using a laboratory EUVL tool at Sandia National Laboratories in Livermore, California. ${ }^{3}$ The EUVL tool uses plasma black-body radiation with a peak at $13.4 \mathrm{~nm}$ generated by focusing a 20 Watt, $20 \mathrm{~Hz} \mathrm{Nd-Yag}$ laser beam on a copper wire target. Molybdenum-silicon multilayer coatings deposited on near-normal incidence optics provide high throughput at this wavelength. A $10 \times$ reduction Schwarzschild camera images a reflective microtag mask pattern onto the resist-coated silicon wafer. The microtags presented in this report were exposed in $0.125 \mu \mathrm{m}$ of ZEP 520 photoresist.

\section{Comparison with Alternative Fabrication Techniques}

The same resist could be used with an electron-beam writer to fabricate a phase-only microtag with comparable feature dimensions. Given a beam-modulation frequency of 25 
$\mathrm{MHz}(0.04 \mu \mathrm{s} / \mathrm{pixel})$, a pattern laid out on a $0.005 \mu \mathrm{m}$ grid required by the minimum grating shift of $0.01 \mu \mathrm{m}$, and a $0.01-\mu \mathrm{m}$-diameter spot-size, an e-beam writer would require approximately $2.5 \mathrm{sec}$ to fabricate an individual $80 \mu \mathrm{m} \times 160 \mu \mathrm{m}$ microtag. ${ }^{10}$

In contrast to electron-beam writing techniques, EUVL exposures possess a parallelprocess advantage necessary for cost-effective mass production of microtags. For example, a 4-mirror-camera EUVL tool under development at Sandia will be able to expose multiple microtags within a ring field of view (FOV) of $25 \mathrm{~mm}$ by $2 \mathrm{~mm}$. The exposure time for a 25 $\mathrm{mm} \times 25 \mathrm{~mm}$ area is $1 \mathrm{sec}$. Writing multiple microtags covering the same area with an ebeam requires approximately 33 hours or approximately $10^{5}$ times longer than using the EUVL tool.

\section{Calculated fabrication tolerances by Rigorous Coupled-Wave Analysis}

Higher diffraction efficiency associated with microtag gratings leads directly to a reduced power requirement on a laser readout source. Our interest in determining the grating parameters that maximize diffraction efficiency is motivated by practical considerations involved in the design of an entire microtag-readout system. Finally, we have chosen to calculate fabrication tolerances rather than to derive them experimentally due to the high cost in resources required by an extensive fabrication and characterization effort.

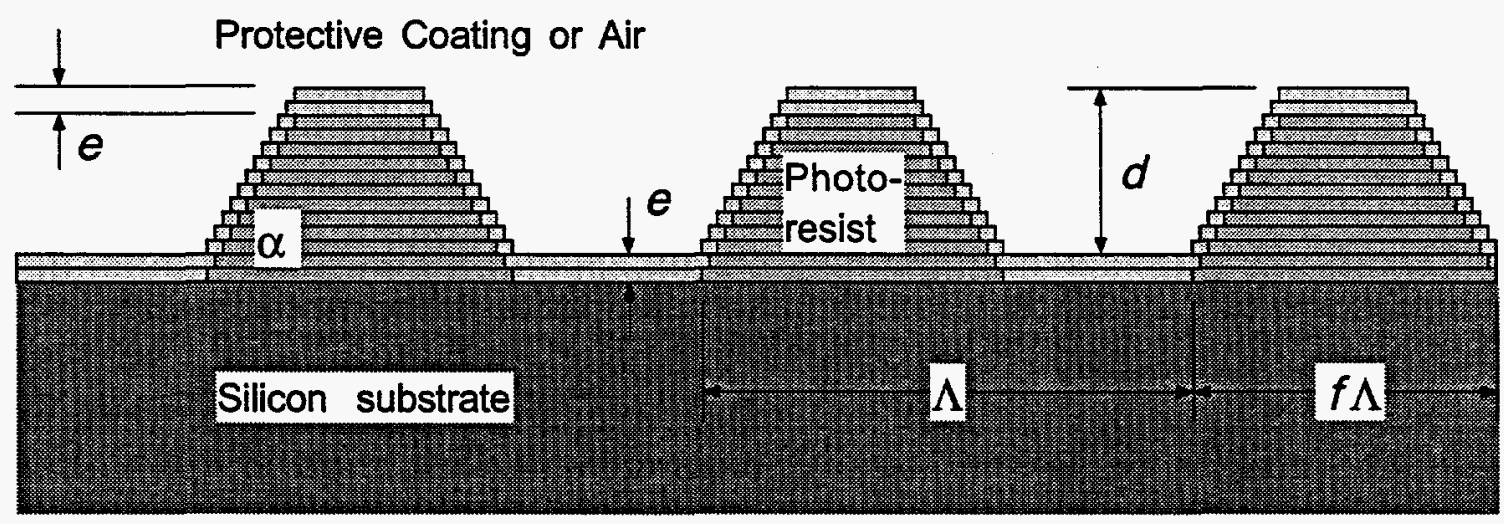

Figure 9. Microtag profile and grating-parameter definition.

\section{Grating Modeling}

Microtags are etched in photoresist deposited on silicon wafers $(n-i k=3.882$ i0.019). We assume that the photoresist is a dielectric medium with an index of refraction of $n_{2}=1.7$. Microtags are assumed to possess a trapezoidal profile, as shown in Figs. 1(a) and 9. The period of microtag gratings is $0.4 \mu \mathrm{m}$. The nominal readout wavelength is $0.6328 \mu \mathrm{m}$ (HeNe laser). The incident medium is either air $\left(n_{1}=1\right)$ or a protective-coating material $\left(n_{1}=1.7\right)$. When present, the metallic coating is assumed to be gold with an index of $n-i k$ $=0.166-i 3.15$ and thickness ranging from $1 \mathrm{~nm}$ to $25 \mathrm{~nm}$. We assume that the metallic 
coating is conformal with the photoresist grating profile, i.e., shifted in the grating-normal direction by the specified coating thickness $e$ (see Fig. 9).

The trapezoidal grating profile is modeled via stratification. We denote the number of strata by the letter $M$. In the case of coated microtags, the stratification is denoted by an array of three values, $[a, b, c]$. These values denote the number of strata in the coating layer thickness $e$ at the substrate, the number of strata in the intermediate (3-material) thickness $d$ $e$, and the number of strata in the top coating-layer thickness $e$, respectively (see Fig. 9).

Only the $(-1)$ st reflected diffraction order is monitored in our calculations. This is the only diffraction order, apart from the $0^{\text {th }}$, supported by the $0.4-\mu \mathrm{m}$ gratings used in each microtag cell. We consider only TE- and TM-polarized read-out-beam illuminations in this study.

\section{Rigorous-Coupled-Wave-Analysis Algorithm Performance Verification}

The algorithm is based on Refs. 11 and 12. We verified the performance of our RCWA code using predictions available in the literature. Table 1 provides a list of literature sources corresponding to five types of gratings. In the case of $\mathrm{TE}$ polarization, the greatest relative error (RE) was found to be $4 \%$ and the least $\mathrm{RE}$ was found to be $4 \cdot 10^{-4} \%$. In the case of TM polarization, the greatest relative error was found to be $15 \%$ and the least RE was found to be $10^{-2} \%$.

\begin{tabular}{lr}
\hline Grating Type/Medium & References \\
\hline Binary/Dielectric & 11 \\
Sinusoidal/Dielectric & 13 \\
Binary/Metal & $14,15,16$ \\
Sinusoidal/Metal & 13 \\
Metal-coated trapezoidal/Dielectric on & 17 \\
Silicon & \\
\hline
\end{tabular}

Table 1. RCWA algorithm verification.

\section{Convergence of the RCWA Algorithm}

We have implemented the modifications of Refs. $16-18$ to improve the RCWA algorithm's convergence in TM and conical-diffraction scenarios. In order to gauge convergence, we used the quantity

$$
\Delta_{N}=-\log _{10}\left|\left(\eta_{i, N}-\eta_{i, N^{\prime}}\right) / \eta_{i, N^{\prime}}\right|
$$

where $\eta_{i, N^{\prime}}$ is the ith diffraction-order efficiency calculated with $N^{\prime}$ terms in the Fourier series expansion of the relative permittivity function in the grating layer, $\eta_{i, N}$ is the $i$ th diffraction-order efficiency calculated with $N$ terms in the Fourier-series expansion of the permittivity, and $N^{\prime} \gg N .{ }^{11,12}$ The magnitude of the quantity defined in Eq. (1) yields the approximate number of converged digits in the modeling results. ${ }^{13}$ We selected two (2) 
converged digits as the target in all calculations or equivalently a relative error of $1 \%$. Based on this target, we determined the number of terms $N$ in the Fourier-series expansion of the relative permittivity in the grating layer: $N=25$ in the case of TE readout polarization $\left(N^{\prime}=\right.$ $105) ; N=75$ in the case of TM readout polarization and bare gratings $\left(N^{\prime}=275\right)$; and $N=$ 165 in the case of TM readout polarization and metal-coated gratings $\left(N^{\prime}=275\right)$. Table 2 presents descriptions of the gratings used in the convergence studies.

\begin{tabular}{|c|c|c|c|c|c|c|}
\hline \multirow[t]{2}{*}{ Polarization } & \multicolumn{2}{|c|}{ TE Polarization } & \multicolumn{4}{|c|}{ TM Polarization } \\
\hline & Grating 1 & Grating 2 & Grating 1 & Grating 2 & Grating 3 & Grating 4 \\
\hline Wall-slope angle & $60^{\circ}$ & $60^{\circ}$ & $60^{\circ}$ & $60^{\circ}$ & $30^{\circ}$ & $80^{\circ}$ \\
\hline Grating duty cycle & 0.5 & 0.5 & 0.5 & 0.5 & 0.5 & 0.5 \\
\hline $\begin{array}{l}\text { Grating period } \\
(\mu \mathrm{m})\end{array}$ & 0.4 & 0.4 & 0.4 & 0.4 & 0.4 & 0.4 \\
\hline Grating depth $(\mu \mathrm{m})$ & 0.05 & 0.05 & 0.05 & 0.05 & 0.05 & 0.05 \\
\hline $\begin{array}{l}\text { Metallic coating } \\
\text { thickness }(\mu \mathrm{m})\end{array}$ & 0.0075 & 0.0075 & 0.0075 & 0.0075 & 0.0075 & 0.0075 \\
\hline $\begin{array}{l}\text { lncident medium } \\
\text { index }\end{array}$ & 11 & 1.7 & 1 & 1.7 & 1 & 1 \\
\hline $\begin{array}{l}\text { Readout-beam } \\
\text { angle of incidence }\end{array}$ & $60^{\circ}$ & $30.625^{\circ}$ & $60^{\circ}$ & $30.625^{\circ}$ & $60^{\circ}$ & $60^{\circ}$ \\
\hline $\begin{array}{l}\text { Readout } \\
\text { wavelength }(\mu \mathrm{m})\end{array}$ & 0.6328 & 0.6328 & 0.6328 & 0.6328 & 0.6328 & 0.6328 \\
\hline
\end{tabular}

Table 2. Grating parameters used in the RCWA convergence studies.

\section{Diffraction Efficiency Sensitivity}

The objective of this study is to determine the effects of varying the following grating parameters: (a) the wall-slope angle, $\alpha$, (b) the depth, $d$, (c) the duty cycle, $f$, and (d) the metallic coating thickness, $e$. All of these parameters are illustrated in Figure 9. Table 3 lists the ranges over which these parameters are varied. Only one of these four parameters can be controlled on a cell-by-cell basis; this is the grating duty cycle. The other parameters are common to the entire microtag. The duty cycle may therefore be used to alter the diffraction efficiency and thus the effective reflectance amplitude of each microtag cell. ${ }^{6}$

\begin{tabular}{llr}
\hline Parameter & Parameter Description (units) & Parameter Range \\
\hline$\alpha$ & wall-slope angle $\left(^{\circ}\right)$ & $30-(80) 110$ \\
\hline$d$ & grating depth $(\mathrm{nm})$ & $30-100$ \\
$f$ & grating duty-cycle (dimensionless) & $0.3-0.8$ \\
$e$ & metallic coating thickness (nm) & $1-25$ \\
\hline no symbol & readout-beam polarization $\left({ }^{\circ}\right)$ & TE, TM \\
\hline
\end{tabular}

Table 3. Microtag parameter nomenclature and variation ranges. The maximum wall-slope angle is $80^{\circ}$ for metal-coated gratings and is $110^{\circ}$ for bare gratings. 
The nominal grating configuration is described in Table 4. In each of the following sections, one of these parameters is varied over the range stated in Table 3 and the results are plotted in terms of the $(-1)$ st order diffraction efficiency.

\begin{tabular}{lr}
\hline Grating Parameter & Nominal Value \\
\hline Wall-slope angle $\alpha\left(^{\circ}\right)$ & 60 \\
Grating duty cycle, $f$ & 0.5 \\
Grating period $\Lambda(\mu \mathrm{m})$ & 0.4 \\
Readout beam angle of incidence $\left({ }^{\circ}\right)$ & 60 \\
Grating depth $d(\mu \mathrm{m})$ & 0.05 \\
Metallic coating thickness $e(\mu \mathrm{m})$ & 0 (bare grating) \\
Readout wavelength $(\mu \mathrm{m})$ & 0.0075 \\
\hline
\end{tabular}

Table 4. Nominal grating parameters.

Note that the readout-beam angle of incidence does not correspond to the Littrow configuration, $\theta=\sin ^{-1}(-\lambda / 2 \Lambda)=-52.3^{\circ}$. Instead, the readout angle $\theta$ has been arranged to separate the incident readout beam and the $(-1)$ st order diffracted output beam. As a result, the $(-1)$ st order output beam is separated from the readout beam by $15^{\circ}$ in air.

\section{Modeling of a protective coating}

A practical implementation of the microtags will most likely require the application of a protective coating. Such a coating is analogous to that used on compact discs. The protective plastic coating on CDs measures $10-30 \mu \mathrm{m}$ in thickness on the printed side of the disc and nearly $1.2 \mathrm{~mm}$ on the readout side. ${ }^{4}$ We can approximate a similar coating by spinning on a layer of photoresist on top of metal-coated microtags. The photoresist used in this way is modeled as a dielectric with $n_{1}=1.7$. The dielectric protective coating will cause reflection losses at the air-protective-coating interface. We calculate the transmissivity associated with the readout beam and estimate the transmissivity associated with the output beam based on

$$
\begin{gathered}
\mathrm{T}_{\mathrm{TE}}=\frac{\sin 2 \theta_{1} \sin 2 \theta_{2}}{\sin ^{2}\left(\theta_{1}+\theta_{2}\right)}, \\
\mathrm{T}_{\mathrm{TM}}=\frac{\sin 2 \theta_{1} \sin 2 \theta_{2}}{\sin ^{2}\left(\theta_{1}+\theta_{2}\right) \cos ^{2}\left(\theta_{1}-\theta_{2}\right)},
\end{gathered}
$$

where $\theta_{1}$ represents the incident angle and $\theta_{2}$ represents the angle after refraction at the interface. ${ }^{19}$ See Fig. 1 for the beams' definition. The output-beam transmissivity is only an 
estimate because it is calculated for one propagation angle following diffraction at the microtag. The calculated transmissivities associated with TE- and TM-polarized readout beams are listed in Table 5. The total transmissivities, $\mathrm{T}_{\mathrm{TE} \text {,total }}$ and $\mathrm{T}_{\mathrm{TM} \text {,total }}$, are accounted for in Figs. 10(b), 11(b), 12(b), and 13(b).

\begin{tabular}{lccc}
\hline Polarization & $\mathrm{T}_{\text {in }}$ & $\mathrm{T}_{\text {our }}$ & $\mathrm{T}_{\text {total }}$ \\
\hline $\mathrm{TE}$ & 0.76 & 0.86 & 0.65 \\
$\mathrm{TM}$ & 0.99 & 0.98 & 0.98 \\
\hline
\end{tabular}

Table 5. Air-protective-coating interface transmissivities.

\section{Wall-slope Angle Variation}

The tolerance on grating wall-slope angle $\alpha$ is different under TE and TM readout polarizations. We calculated the $(-1)$ st reflected diffraction order efficiency under the nominal configuration of Table 3 while varying the angle $\alpha$. We terminated the diffractionefficiency plots for metal-coated gratings in Fig. 10 at $80^{\circ}$. The conformal-coating assumption taken earlier is doubtful at higher wall-slope angles. The diffraction-efficiency plots for bare gratings continue up to $110^{\circ}$ in an effort to model negative resists that can be expected to have an "undercut" profile. Recall that wall slope angle cannot be varied across the microtag.

Figure 10(a) indicates that the wall-slope angle has the most influence on diffraction efficiency of metal-coated gratings under TM illumination. Curves (a) and (b) in the same Figure show that under TE illumination, on the other hand, the wall-slope angle does not significantly affect $\eta_{-1}$. Similarly, the diffraction efficiency of bare gratings under TM illumination is not significantly influenced by wall-slope angle. Figure 10(a) also shows that higher diffraction efficiency is obtained under TM illumination than under TE illumination and that the highest diffraction efficiency occurs in the case of metal-coated gratings. Table 7 quantifies these observations. The tolerances in Table 7 are defined as the wall-slope-angle ranges over which the diffraction efficiency exceeds $90 \%$ of its peak value. In case of metalcoated gratings under TE illumination, diffraction efficiency continues to rise with increasing wall-slope angle. Because of this trend, the diffraction efficiency at $80^{\circ}$ is the de facto peak diffraction efficiency.

\begin{tabular}{lcccc|ccc}
\hline Figure & \multicolumn{3}{c|}{ 10(a), 11(a), 12(a) } & \multicolumn{3}{c}{ 10(b), 11(b), 12(b) } \\
\hline Plot & (a) & (b) & (c) & (d) & (a) & (b) \\
\hline Readout Polarization & TE & TE & TM & TM & TE & TM \\
Metal coating & Yes & No & Yes & No & Yes & Yes \\
Incident medium index & 1 & 1 & 1 & 1 & 1.7 & 1.7 \\
\hline
\end{tabular}

Table 6. Legend for Figures 10-12. 


\begin{tabular}{|c|c|c|c|c|c|c|}
\hline \multirow[t]{2}{*}{ Grating Type } & \multicolumn{2}{|c|}{ Peak Diffraction Efficiency } & \multicolumn{2}{|c|}{$\begin{array}{c}\text { Peak-diffraction-efficiency } \\
\text { Wall-slope Angle }\end{array}$} & \multicolumn{2}{|c|}{$\begin{array}{c}\text { Wall-slope Angle } \\
\text { Tolerance }\end{array}$} \\
\hline & $\mathrm{TE}$ & TM & $\mathrm{TE}$ & TM & TE & TM \\
\hline Bare & 0.0013 & 0.014 & $92^{\circ}$ & $62^{\circ}$ & $63^{\circ}-110^{\circ \dagger}$ & $40^{\circ}-102^{\circ}$ \\
\hline Metal-coated & 0.0030 & $0.033+1 \%$ & $80^{\circ}$ & $44^{\circ}$ & $51^{\circ}-80^{\circ}$ & $35^{\circ}-49^{\circ}$ \\
\hline $\begin{array}{l}\text { Protective- } \\
\text { coating }^{\ddagger}\end{array}$ & 0.010 & $0.013 \pm 1 \%$ & $80^{\circ}$ & $54^{\circ}$ & $55^{\circ}-80^{\circ \dagger}$ & $48^{\circ}-58^{\circ}$ \\
\hline
\end{tabular}

Table 7. Wall-slope effects on diffraction efficiency $\eta_{-1}$.

${ }^{\dagger}$ These wall-slope angles represent the upper bound on the modeled range.

${ }^{\ddagger} \mathrm{TE}$ and TM transmissivities at the air-protective-coating interface are included in the Table entries.

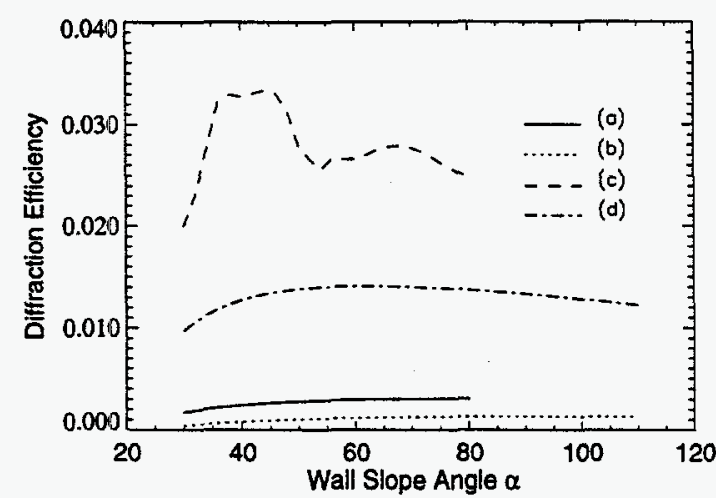

(a)

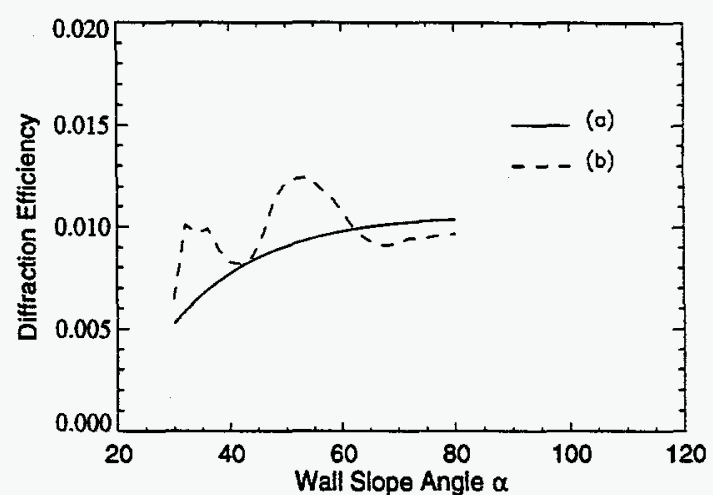

(b)

Figure 10. Sensitivity of the (-1)st order diffraction efficiency to grating wall-slope angle. Note the change in the ordinate maximum between (a) and (b). See Table 6 for legend.

Figure 10(b) shows the effect of wall-slope angle on metal-coated microtags under a protective dielectric coating. The highest diffraction efficiency is associated with TM illumination. The effect of wall-slope angle on $\eta_{-1}$ is more pronounced under this polarization as well. The tolerance on wall-slope angle is approximately half as wide under TM illumination as it is under TE illumination (see Table 7).

\section{Depth variation}

The effect of grating depth on diffraction efficiency can be summarized by the statement: Deeper is better. The only exception to this rule is the case of a metal-coated grating under a protective dielectric layer under TM illumination [Curve (b) in Fig. 11(b)]. In that particular case, the highest diffraction efficiency of $\eta_{-1}=0.023$ is reached at a grating depth of $0.078 \mu \mathrm{m}$. The corresponding tolerance on the grating depth is $0.069-0.084 \mu \mathrm{m}$. We expect a maximum grating depth of $0.1 \mu \mathrm{m}$ and that depths between 0.06 and $0.08 \mu \mathrm{m}$ 
are within easy reach, depending on photoresist. The depth limitation is imposed by photoresist absorption. For reference, note that the pit depth found on a compact disc is approximately $0.11 \mu \mathrm{m} .{ }^{4}$ Finally, recall that the grating depth is similar to the wall-slope angle because it cannot be altered across a microtag.

Figure 11 extends the above discussion by showing the sensitivity of the $(-1)$ st order diffraction efficiency to grating depth. In Figure 11(a), bare and metal-coated gratings are modeled under TE and TM polarizations and air as the incident medium. In Figure 11(b), the diffraction efficiency for metal-coated gratings under a protective dielectric coating and both polarizations is shown. See Table 6 for a legend corresponding to Fig. 11. All grating parameters except the grating depth, $d$, are as listed in Table 3.

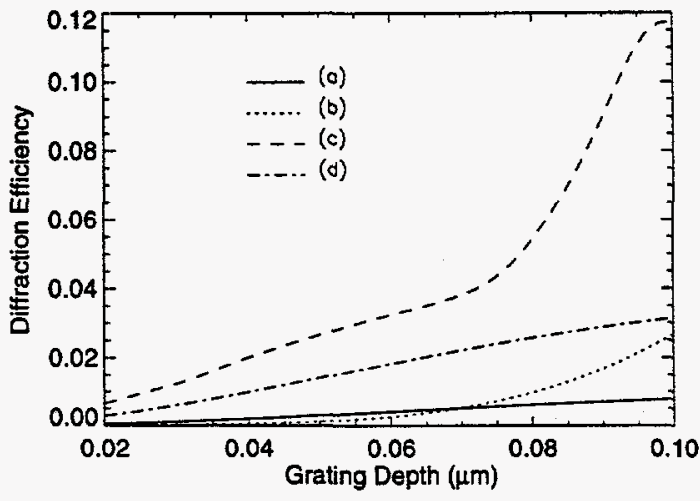

(a)

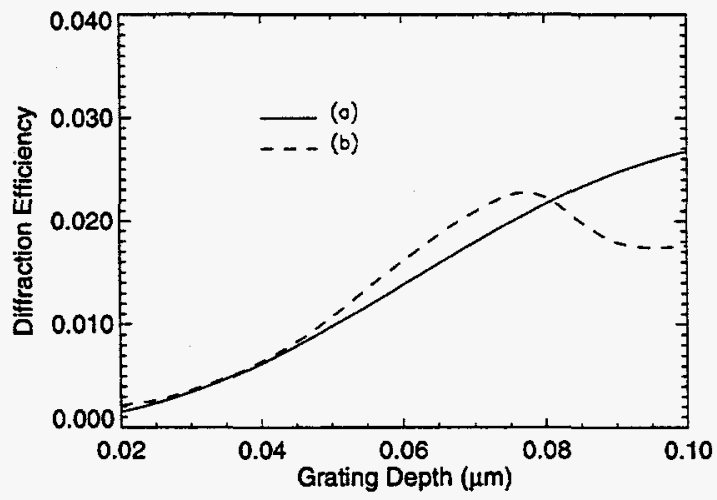

(b)

Figure 11. Sensitivity of the $(-1)$ st order diffraction efficiency to grating groove depth. Note the change in the ordinate maximum between (a) and (b). See Table 6 for legend.

In the absence of a protective coating, a TM-polarized readout beam is diffracted with the highest efficiency, $\eta_{-1}=0.11$, at a grating depth $d=0.1 \mu \mathrm{m}$. The most significant gains in diffraction efficiency occur for grating depths $d>0.08 \mu \mathrm{m}$ [Curve (c) in Fig. 11(a)]. In the presence of a protective coating, the highest diffraction efficiency is associated with a TEpolarized readout beam, $\eta_{-1}=0.027$, at a grating depth $d=0.1 \mu \mathrm{m}$. In contrast to all other cases considered in this Section, the peak diffraction efficiency associated with a TMpolarized readout beam occurs at an intermediate grating depth, already mentioned above.

\section{Duty-cycle variation}

The grating duty cycle is a unique parameter because it can be varied across the microtag. Specifically, duty cycle may be used to adjust the reflectance amplitude within each microtag cell. ${ }^{6}$ It is a simple matter to modify the width of the grating features in each cell at the photomask-layout stage. In addition, a modification of the duty cycle may result in a different change in amplitude reflectance under TM versus TE illumination. This property can be used to introduce polarization sensitivity into the amplitude encoding within a microtag. 
In the calculation of Fig. 12, all grating parameters with the exception of grating duty cycle, $f$, were held at the values listed in Table 4. The optimal, i.e., peak-diffractionefficiency, grating duty cycles for each microtag-grating type are listed in Table 8. The dutycycle tolerance is again bounded by values of $f$ that correspond to diffraction efficiencies equal to $90 \%$ of the peak value. Double entries are a result of the tolerance definition and the dual peaks in curves 12(a)(c) and 12(b)(b). The duty cycle tolerance for microtags with a protective coating is practically the same for TE and TM readout polarizations.

\begin{tabular}{|c|c|c|c|c|c|c|}
\hline \multirow[t]{2}{*}{ Grating Type } & \multicolumn{2}{|c|}{$\begin{array}{l}\text { Peak Diffraction } \\
\text { Efficiency }\end{array}$} & \multicolumn{2}{|c|}{$\begin{array}{c}\text { Peak-diffraction-efficiency } \\
\text { Duty Cycle }\end{array}$} & \multicolumn{2}{|c|}{ Duty Cycle Tolerance } \\
\hline & $\mathrm{TE}$ & $\mathrm{TM}$ & TE & $\mathrm{TM}$ & $\mathrm{TE}$ & $\mathrm{TM}$ \\
\hline Bare & 0.0013 & 0.014 & 0.58 & 0.48 & $0.49-0.69$ & $0.37-0.58$ \\
\hline Metal-coated & 0.0030 & $\begin{array}{l}0.028 \pm 1 \% \\
0.029+1 \%\end{array}$ & 0.53 & $\begin{array}{l}0.38 \\
0.54\end{array}$ & $0.44-0.64$ & $\begin{array}{l}0.35-0.41 \\
0.49-0.63\end{array}$ \\
\hline Protective- & 0.010 & $0.011 \pm 1 \%$ & 0.56 & 0.46 & $0.47-0.67$ & $0.42-0.51$ \\
\hline coating $^{\ddagger}$ & & $0.011 \pm 1 \%$ & & 0.62 & & $0.58-0.67$ \\
\hline
\end{tabular}

Table 8. Duty-cycle effects on diffraction efficiency $\eta_{-1}$. Double entries correspond to double peaks in the plots of Figure 12.

${ }^{\ddagger} \mathrm{TE}$ and TM transmissivities at the air-protective-coating interface are included in the Table entries.

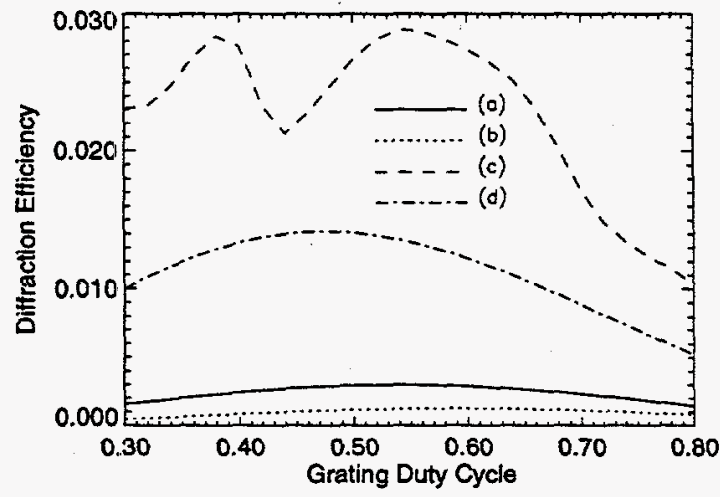

(a)

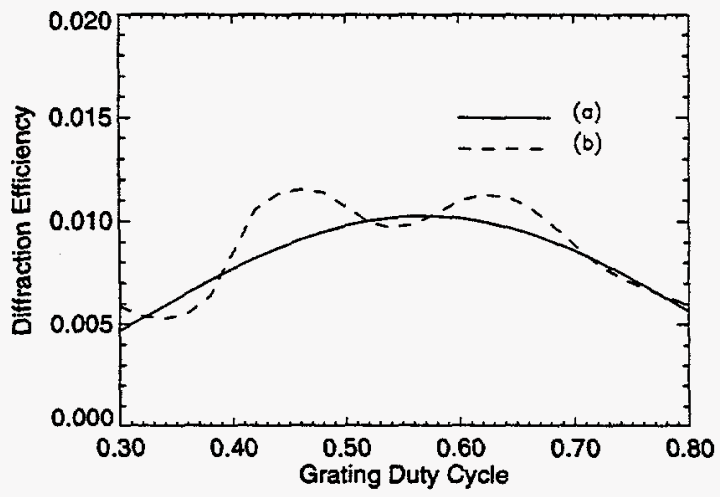

(b)

Figure 12. Sensitivity of the (-1)st order diffraction efficiency to grating duty cycle, $f$. Note that the duty cycle is measured at the base of the grating, i.e., at the silicon substrate. Note the change in the ordinate maximum between (a) and (b). See Table 6 for legend.

\section{Effect of Metallic Coating Thickness}

The effect of varying the metallic coating thickness is similar to that observed by varying the depth of microtag gratings. In this case, thicker is better. 
Recall that the metallic coating is assumed to be conformal with the underlying dielectric grating profile. We have only considered metal-coating thicknesses up to $25 \mathrm{~nm}$. A metal-coating thickness of $25 \mathrm{~nm}$ corresponds to one-half of the nominal grating depth (see Table 4). Beyond this maximum thickness, the conformal-profile assumption may need to be examined. Figure 13 shows that a thicker metallic coating leads to higher diffraction efficiency (see Table 9 for associated legend). In modeling the effects of the metallic-coating thickness, a stratification of $[5,10,5]$ was adopted (see Fig. 9). Finally, for reference consider again the compact-disc system. Compact-disc pits are coated with a layer of metal (silver, gold, or aluminum) of thickness between 50 and $100 \mathrm{~nm}^{4}$

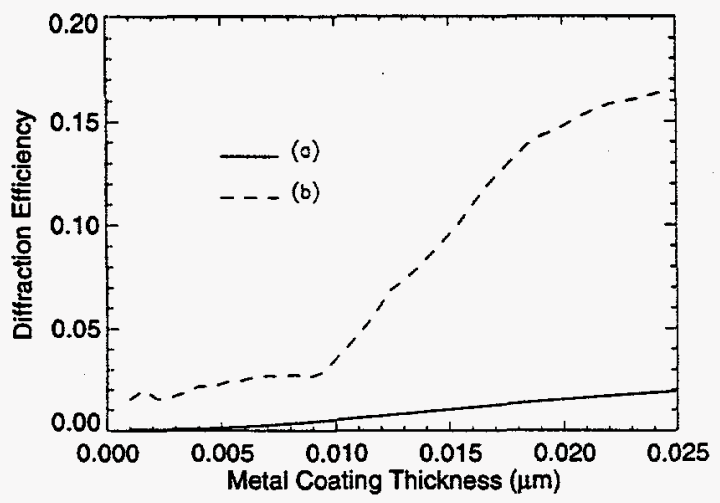

(a)

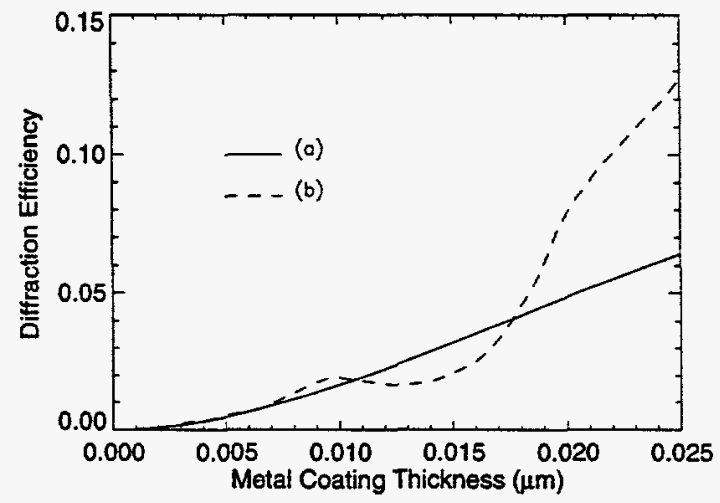

(b)

Figure 13. Effect of metal-coating thickness on read-out diffraction efficiency. Note the change in the ordinate maximum between (a) and (b). See Table 9 for legend.

\begin{tabular}{lcc|cc}
\hline Figure & \multicolumn{2}{c|}{$\mathbf{8}(\mathbf{a})$} & \multicolumn{2}{c}{$\mathbf{8}(\mathbf{b})$} \\
\cline { 2 - 6 } Plot & (a) & (b) & (a) & (b) \\
\hline Readout Polarization & TE & TM & TE & TM \\
$\begin{array}{l}\text { Metal coating } \\
\begin{array}{l}\text { Incident medium } \\
\text { index }\end{array}\end{array}$ & Yes & Yes & Yes & Yes \\
\hline
\end{tabular}

Table 9. Legend for Figure 13.

In air and under TM illumination, the application of a metallic coating leads to a maximum (-1)st order diffraction efficiency of $0.16[e=0.025 \mu \mathrm{m}$ in Fig. 9(a)]. The (-1)storder diffraction efficiency is not sensitive to metallic coating thickness for $e<10 \mathrm{~nm}$. For example, increasing the thickness from 5.2 to $8.8 \mathrm{~nm}$ leads to only a $10 \%$ increase in $\eta_{-1}$. In contrast, increasing the thickness by the same $\Delta e=3.6 \mathrm{~nm}$, from 10 to $13.6 \mathrm{~nm}$, leads to a 
$125 \%$ increase in $\eta_{-1}$. Between the metal thicknesses of $10 \mathrm{~nm}$ and $18 \mathrm{~nm}$, the diffraction efficiency increases 0.012 for every nanometer of deposited metal. Under TE illumination, diffraction efficiency does increase with increasing metallic coating thickness, but lags the efficiency associated with TM illumination by a wide margin [Fig. 8(a)].

In the presence of a protective coating, the maximum diffraction efficiency of 0.12 is again achieved under TM illumination and at the maximum modeled coating thickness $(0.025$ $\mu \mathrm{m})$.

Diffraction efficiencies achieved by increasing the metallic coating thickness are rivaled only by the results of increasing grating depth. For example, $\eta_{-1}=0.11$ for a 0.1 micron deep metal coated grating in air under TM illumination [Fig. 6(a)]. In all other cases, the maximum diffraction efficiencies do not exceed 0.033 (see Tables 7 and 8 ). Furthermore, the deposition of a metallic coating is much easier to carry out. For instance, a metallic coating of up to 80-nm thick can be deposited in under five minutes. The metallic coating is therefore the most effective means of maximizing the diffraction efficiency of microtag gratings.

\section{Additional Microtag Concepts}

\section{Two-dimensional Microtags}

A standard feature of the microtag designs discussed in the previous sections is that each microtag forms only one diffraction pattern. Can a microtag be designed to form multiple and distinct diffraction patterns? Under what conditions could this objective be achieved?

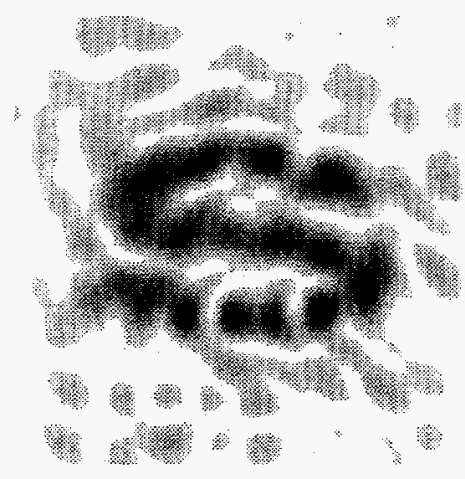

(a)

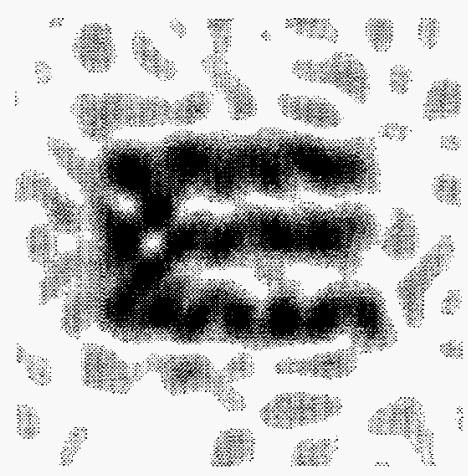

(b)

Figure 14. Predicted diffraction pattern for a $2 \mathrm{D}$ phase-only microtag.

If two distinct diffraction patterns are to be obtained from a single microtag then effectively two microtags need to be combined within the same $80 \mu \mathrm{m} \times 80 \mu \mathrm{m}$ area. Each microtag needs to be invisible to the other in order to avoid cross-talk between the two diffraction patterns. Recall that phase at each microtag cell is encoded via a shift of that cell's grating in a direction perpendicular to the grating lines (see Fig. 14). If the single grating within each cell is replaced instead by a crossed grating and the grating translations 
are applied in the $x$ and $y$ directions then two separate microtags can be combined within the same area. During readout, the microtag is first oriented to form one of the diffraction patterns, say Fig. 14(a). Second, the microtag is rotated $90^{\circ}$ in its plane and the second diffraction pattern is formed, Fig. 14(b).

The diffraction patterns shown in Fig. 14 are compressed in the vertical direction for a reason. The $2 \mathrm{D}$ microtag and its cells will appear rectangular in the direction of the (-1)st diffraction order. The diffraction patterns will therefore become elongated in the vertical direction (in the sense of Fig. 14). In order to compensate for this effect, the desired diffraction pattern is therefore compressed prior to designing the microtag.

\section{Pixel-code Microtags}

Another feature common to the microtags discussed in previous sections is that the diffraction pattern is in the shape of the letter $\mathbf{E}$. Such a diffraction pattern is a special case of a more general collection; that of alpha-numeric characters (A-Z, $0-9)$. Selection of alphanumeric-character patterns is based on the implicit assumption that a human observer examines the microtag diffraction pattern. In some applications, however, automated recording and recognition of the diffraction patterns may be preferred. Alpha-numeric characters may no longer be optimal. An alternative is shown in Figure 15. The diffraction patterns shown in Fig. 15 consist of 49 pixels. Each pixel can be turned on or off $(1$ or 0$)$. In principle, there are $2^{49}$ or $5.6 \times 10^{14}$ distinct patterns that can be encoded in a given microtag.

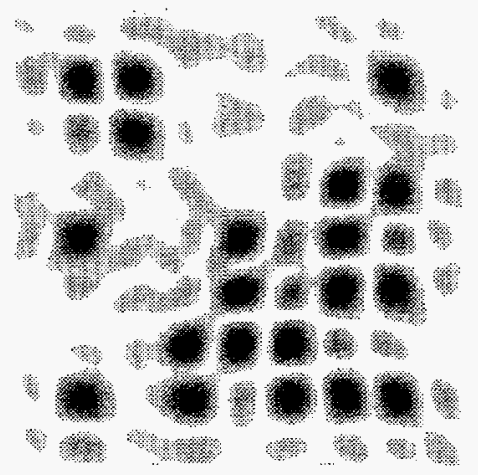

(a)

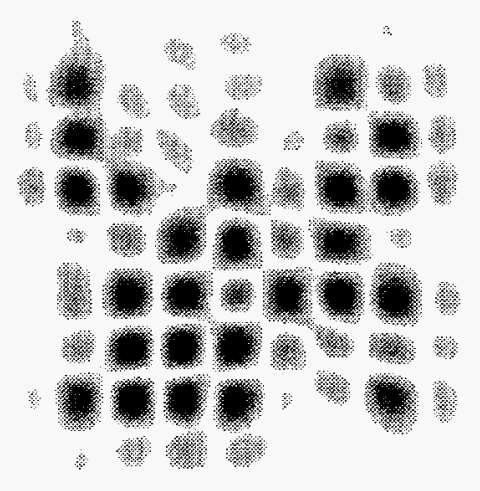

(b)

Figure 15. Predicted diffraction patterns for a "pixel-code" phase-and-amplitude microtag.

\section{"Continuous" Phase-and-amplitude Microtags}

A limitation common to all microtags described in this report is the 0.1 -micron spotsize and positioning accuracy of the electron-beam writer that writes the photomask. If the microtag photomask is written with a smaller-spot electron beam on a finer position grid, the number of phase-quantization levels and amplitude levels can be increased. Such a photomask has been written using a $0.025-\mu \mathrm{m}$ spot and position grid. The microtags on this photomask consist of gratings with a $3-\mu \mathrm{m}$ period. As a result, 120 equally spaced phase- 
quantization levels could be used in the microtag design process. Similarly, the number of amplitude levels was increased from 10 to 40 . Figure 16 shows the predicted diffraction pattern for this "continuous" phase-and-amplitude microtag. Compare the diffraction pattern shown in Fig. 16 with the predicted diffraction pattern shown in Fig. 5(a). The nearly continuous phase and amplitude values allow for the suppression of speckle features within the letter $\mathbf{E}$.

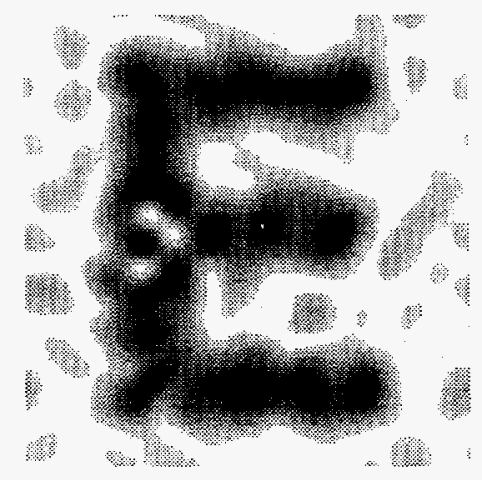

Figure 16. Predicted diffraction pattern for a 120 phase-quantization level and 40 amplitudelevel microtag.

\section{Practical Implementation}

In a practical implementation, microtags will benefit from embedding within a protective transparent layer of plastic, such as polycarbonate. This layer will reduce the effect of contaminants and scratches on the surface of a card or a disc during readout. A plastic layer will also render non-destructive access to microtags very difficult. Highfrequency lamellar gratings embedded in a transparent medium and used in the $0^{\text {th }}$ order have been already been suggested by Gale, et al., as a security measure. ${ }^{20}$ The grating period discussed in their paper was $0.4 \mu \mathrm{m}$. Such sub-wavelength-period, embedded gratings exhibit very distinct polarization properties depending on the viewing angle. 


\section{Conclusions and Further Work}

We have described a method for encoding phase and amplitude in microscopic diffractive computer-generated holograms or microtags, for short. Phase and amplitude are encoded by means of translation and trimming of sub-wavelength-period gratings (see Fig. 2). Microtags were developed as security and anti-counterfeiting devices.

Figures 2(b) and 3(b) illustrate the diffraction patterns formed by 400 -nm-periodgrating microtags formed at $632.8 \mathrm{~nm}$. These diffraction patterns match their predicted counterparts extremely well [compare Figs. 2(a) and 2(b) and Figs. 3(a) and 3(b)]. The agreement is satisfactory in terms of the reproduction of the letter " $E$ " and the reproduction of the speckle patterns.

The diffraction patterns formed by the 150-nm-period-grating microtags at $442 \mathrm{~nm}$ and the corresponding predicted diffraction patterns are also in very good agreement. Figures 5(b) and 6(b) each contain all the major detail present in the predicted patterns shown in Figs. 5(a) and 6(a), respectively. Also note again that even the more subtle speckle features appear as predicted.

We have applied RCWA to microtag gratings to establish tolerances on the fabrication of these diffractive elements. We considered two types of parameters; those that vary across the microtag by design (duty cycle, $f$ ) and those that are uniform across the microtag (wall-slope angle, grating depth, metallic-coating thickness). The fabrication tolerances were established by treating grating parameters individually. A nominal grating configuration was defined (Table 4) and $\eta_{-1}$ was computed vs. one grating parameter at a time. Tolerances were derived from parameter ranges over which $\eta_{-1} \geq 0.9\left(\eta_{-1}\right)_{\max }$.

If air is the incident medium, i.e., microtags are surface-relief patterns on a suitable substrate, then the preferred readout-beam polarization is TM. This preference is dictated by $(-1)$ st-order diffraction efficiency being higher at this polarization than at TE. Increasing the grating depth and increasing the metallic coating thickness both lead to maximum values of $\eta_{-1}, 0.11$ and 0.16 , respectively (see Sections 3.2 and 3.4). The higher maximum diffraction efficiency associated with metal coating and the relative ease of applying it make it the preferred method of increasing diffraction efficiency.

If a protective coating is used, i.e., microtags consist of a thin metal layer sandwiched between two dielectric layers, TM is the preferred read-out polarization under most conditions. There are several cases where TE-polarized readout illumination is diffracted with higher efficiency than TM-polarized readout illumination. Specifically, these cases are: (1) wall-slope angle $\alpha>62^{\circ}$ [Fig. 10(b)], (2) grating depth $d>0.081 \mu \mathrm{m}$ [Fig. 11(b)], (3) duty cycle $0.52<f<0.56$, and (4) metallic coating thickness $0.011 \mu \mathrm{m}<e<0.017 \mu \mathrm{m}$ [Fig. 13(b)]. In all cases except that of grating depth, the highest diffraction efficiency is associated with TM readout polarization. 


\section{APPENDIX A. Design Workshop 2000 Macro for Microtag Photomask Layout}

Microtag photomasks are laid out using the dw2000 software from Design Workshop. Recall that the phase and amplitude values determined by the microtag design algorithm (Gerchberg-Saxton and Perturbation Method) need to be encoded in the microtag pattern. The encoding of phase values is implemented through grating shifts. The encoding of amplitude values is implemented through trimming the grating width (see Fig. 2).

The dw2000 macro listed below is representative of the layout of a phase-andamplitude microtag. Comments are dispersed throughout to help the reader gain an understanding for the macro's function.

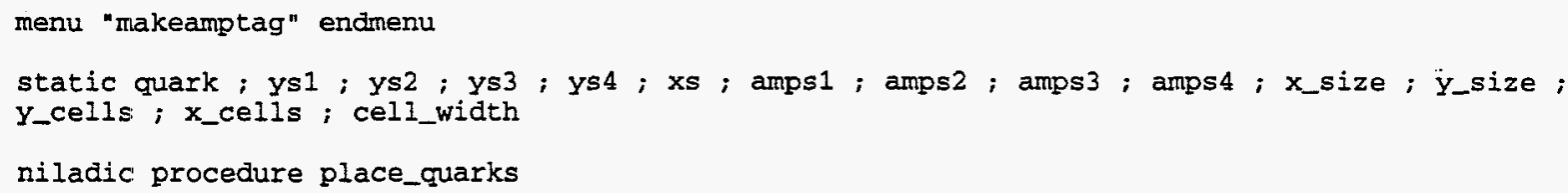

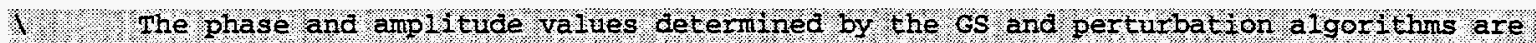

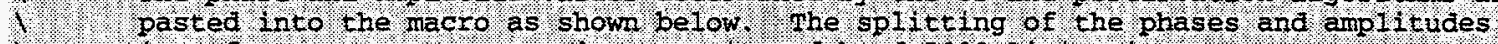

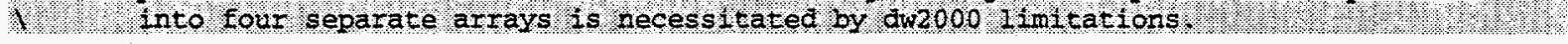

Ivalues from lotsophe.amp

amps $1:=0.000,0.000,0.000,0.250,0.350,0.250,0.225,0.400,0.000,0.300,0.175,0.000^{\wedge}$ $0.125,0.175,0.250,0.425,0.475,0.000,0.000,0.450,0.575,0.000,0.325,0.150^{\wedge}$ $0.100,0.125,0.200,0.450,0.350,0.300,0.500,0.350,0.400,0.250,0.325,0.200^{\wedge}$

amps $2:=0.175,0.325,0.325,0.450,0.625,0.500,0.000,1.000,0.650,0.450,0.000,0.100^{\wedge}$ $0.125,0.050,0.250,0.350,1.000,0.875,0.475,0.650,0.350,0.850,0.200,0.000^{\wedge}$ $0.125,0.175,0.375,0.375,0.025,0.325,0.300,0.000,0.125,0.175,0.150,0.050^{\wedge}$

$\operatorname{amps} 3:=0.075,0.075,0.000,0.000,0.175,0.000,0.525,0.600,0.375,0.500,0.325,0.175^{\wedge}$ $0.100,0.300,0.325,0.475,0.600,0.375,0.050,0.475,0.675,0.300,0.000,0.175^{\wedge}$ $0.000,0.150,0.250,0.350,0.150,0.200,0.350,0.175,0.300,0.250,0.025,0.000^{\wedge}$

$\operatorname{amps} 4:=0.000,0.100,0.075,0.000,0.150,0.175,0.000,0.350,0.000,0.275,0.000,0.100 \wedge$ $0.125,0.250,0.225,0.250,0.200,0.175,0.000,0.000,0.325,0.125,0.075,0.075^{\wedge}$ $0.100,0.125,0.000,0.000,0.075,0.125,0.000,0.125,0.000,0.150,0.050,0.075^{\wedge}$

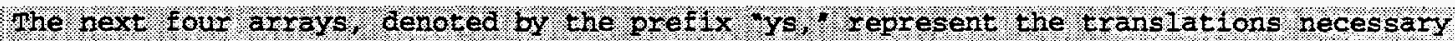

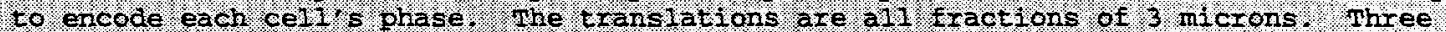

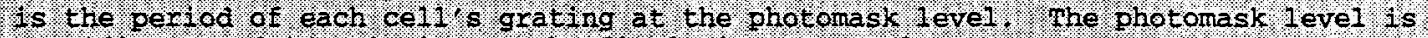

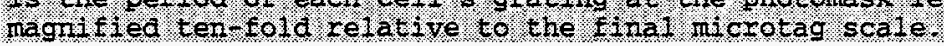

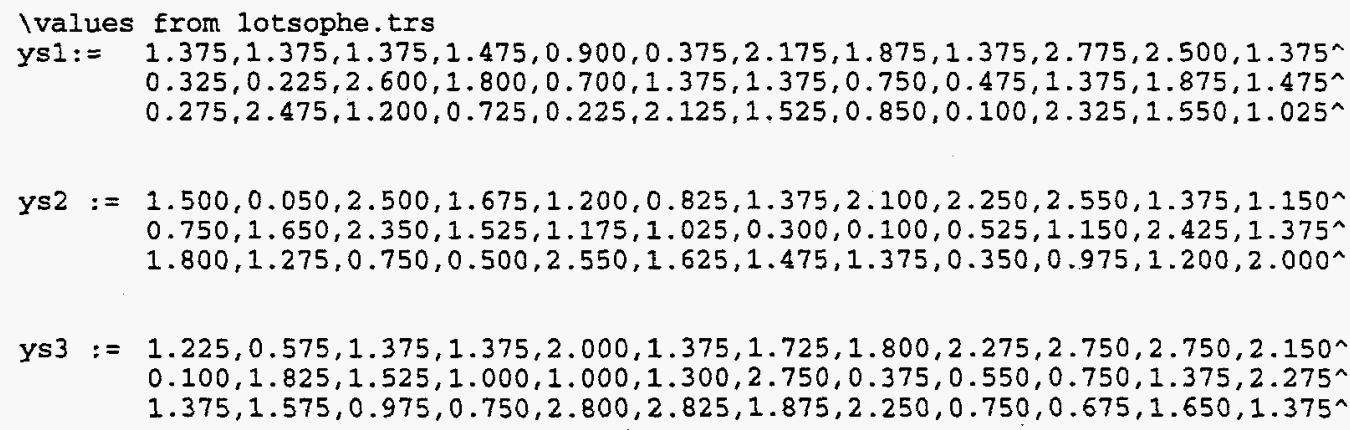


ys4 $:=1.375,2.175,0.075,1.375,1.375,1.825,1.375,1.725,1.375,2.775,1.375,2.050^{\wedge}$ $0.000,1.800,2.200,0.900,0.150,1.625,1.375,1.375,0.550,1.475,2.825,0.200^{\wedge}$ $1.850,1.600,1.375,1.375,0.450,2.800,1.375,1.425,1.375,2.225,2.450,2.100^{\wedge}$

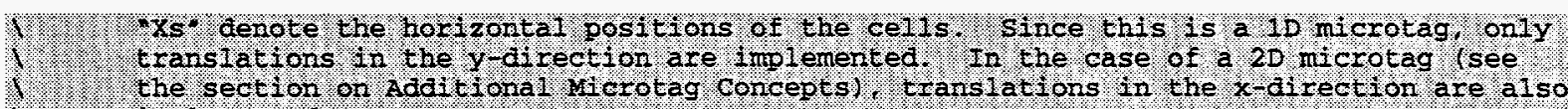
1. Implemented.

xs $:=0.70 .140 .210 .280 .350 .420 .490 .560 .630 .700 .770$.

x_size $:=70$.

Y_size $:=102$.

x_cells $:=12$

$y_{-}$cells $:=3$

cell_width $:=1.75$

endsub

niladic procedure makeamptag

local $i ; j ; k ; m ; x_{0} ; x_{1} ; y 0 ;$ amplitude

local integer widel ; wide2

place_quarks

SREF

SNAME quark

Layer 1

1. The same macro sequence is repeated four times, to handle the four separate

1. amplitude and phase arrays. Amplifude is encoded not as a single wide grating of a

1. prescribed wideh within each cell but rather as two narrower gratings. The summed.

1. widths of the narrower gratings add up to the fraction of the maximum cell width that

1.2 is the cellis reflectance. YO $:=0$

for $j$ RANGE IOTA $y_{-}$cells DO

for i RANGE IOTA $x$ _cells DO

amplitude $:=\operatorname{amps} 1[i+(j-1) * 12] * x$ size/cell wiath

widel $:=$ amplitude/2 luse widel \& wide2 to break amp

wide2 := amplitude - widel linto 2 evenly spaced blocks

$x 0:=x s[i]+x \_s i z e / 4$. - wide1*cell_width/2

$x 1:=x 0+x_{-} \operatorname{size} / 2$

y0 :=ys1 $[i+(j-1) * 12]+(j-1) * y$ size

if widel $>0$ then

for $k$ RANGE IOTA WIdel DO

$C E \times 0, Y 0$

PUT

ENDDO

$\mathrm{x} 0:=\mathrm{x} 0+$ cell_width

endif

if wide2 $>0$ then

for I RANGE IOTA wide2 DO

CE XI,YO

PUT

ENDDO

$\mathbf{x} 1:=\mathbf{x} 1+$ cell_width

endif

ENDDO

ENDDO

for $j$ RANGE IOTA $Y$ _cells DO

for $i$ RANGE IOTA $x_{-}$cells DO

amplitude $:=$ amps $2[i+(j-1) * 12] * x$ size/cel1_width

widel := amplitude/2 luse widel \& wide2 to break amp

wide2 := amplitude - widel linto 2 evenly spaced blocks

$x 0:=x s[i]+x \_s i z e / 4$. - wide1*cell_width $/ 2$

$x 1:=x 0+x_{-}$size $/ 2$

$y 0:=y s 2[i+(j-1) * 12]+(j-1) * y \_s i z e+3 * y \_s i z e$

if widel $>0$ then

for $k$ RANGE IOTA widel DO

CE $X 0, Y 0$

PUT

$\mathbf{x} 0:=x 0+$ cell_width 


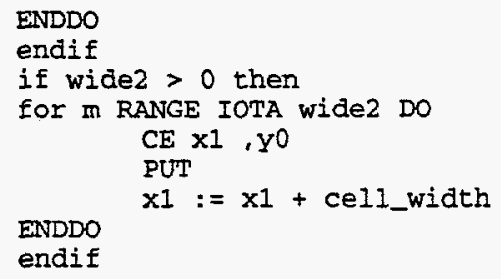




\section{REFERENCES}

W.C. Sweatt, M.R. Descour, A.K. Ray-Chaudhuri, S.H. Kravitz, M.E. Warren, R.H. Stulen, D.A. Tichenor, J.H. Underwood, and K.D. Krenz, in Diffractive and Holographic Optics Technology III SPIE 2689, I. Cindrich, S.H. Lee, eds. (SPIE, Bellingham, WA, February 1996), p. 170.

2 J.L. Zoreda and J.M. Otón, Smart Cards (Artech House, Boston, MA, 1994).

3 D.A. Tichenor, in OSA Proceedings on Extreme Ultraviolet Lithography, F. Zernike and D.T. Attwood, eds., (Optical Society of America, Washington, DC, 1994), p. 89.

4 K.C. Pohlmann, The Compact Disc Handbook, $2^{\text {nd }}$ ed. (A-R Editions, Inc., Madison, WI, 1992).

5 B.R. Brown and A.W. Lohmann, App. Opt., 5, 967 (1966).

6 M. Li, A. Larsson, N. Eriksson, M. Hagberg, and J. Bengtsson, Opt. Lett., 21, 1516 (1996).

$7 \quad$ R.W. Gerchberg and W.O. Saxton, Optik, 35, 237 (1972).

$8 \quad$ P.E. Keller and A.F. Gmitro, App. Opt., 31, 5517 (1992).

9 J.D. Gaskill, Linear Systems, Fourier Transforms, and Optics, (John Wiley \& Sons, New York, 1978), Ch. 10.

10 J.R. Wendt, Sandia National Laboratories, Albuquerque, NM 87185 (personal communication, 1996).

11 M.G. Moharam, E.B. Grann, D.A. Pommet, T.K. Gaylord, "Formulation for stable and efficient implementation of the rigorous coupled-wave analysis of binary gratings,"JOSA $A, 12$, No. 5, pp. 1068-1076 (May 1995).

12 M.G. Moharam, D.A. Pommet, E.B. Grann, T.K. Gaylord, "Stable implementation of the rigorous coupled-wave analysis for surface-relief gratings: enhanced transmittance matrix approach" JOSA A, 12, No. 5, pp. 1077-1086 (May 1995).

13 L. Li, "Multilayer modal method for diffraction gratings of arbitrary profile, depth, and permittivity," JOSA A, 10, No. 12, pp. 2581-2591 (December 1993).

$14 \quad$ L. Li and C.W. Haggans, "Convergence of the coupled-wave method for metallic lamellar diffraction gratings,” JOSA A, 10, No. 6, pp. 1184-1189 (June 1993). 


\section{REFERENCES CONTINUED}

15 P. Lalanne, G.M. Morris, "Highly improved convergence of the coupled-wave method for TM polarization," JOSA A, 13, No. 4, pp. 779-784 (April 1996).

16 G. Granet and B. Guizal, "Efficient implementation of the coupled-wave method for metallic lamellar gratings in TM polarization," JOSA A, 13, No. 5, pp. 1019-1023 (May 1996).

17 L. Li, Optical Sciences Center, University of Arizona, Tucson, AZ 85721 (private communication, 1997).

18 P. Lalanne, G.M. Morris, "Highly improved convergence of the coupled-wave method for TM polarization,” JOSA A, 13, No. 4, pp. 779-784 (April 1996).

19 M. Born and E. Wolf, Principles of Optics, Ch. 1 (Pergamon Press, Oxford, 1980).

20 M.T. Gale, K. Knop, and R. Morf, in Optical security and anticounterfeiting systems, SPIE 1210, W.F. Fagan, ed. (SPIE, Bellingham, WA, 1990), p. 83.

\section{Distribution:}

20 MS 0603 W. C. Sweatt, 01312

$5 \quad$ MS 9161 K. D. Krenz, 08250

2 MS 9161 R. H. Stulen, 08250

1 MS 9161 A. K. Ray-Chaudhuri, 08250

2 MS 9161 D. A. Tichenor, 08250

2 MS 0603 M. E. Warren, 01312

1 MS 0603 S. H. Kravitz, 01313

1 MS 0972 C. A. Boye, 05705

1 MS 0603 J. R. Wendt, 01314

1 MS 0161 Patent and Licensing Office, 11500

1 MS 9018 Central Technical Files, 08940-2

$5 \quad$ MS 0899 Technical Library, 04916

2 MS 0619 Review and Approval Desk, 12690

For DOE/OSTI 